\title{
Inferring Insertion Times and Optimizing Error Penalties in Time-decaying Bloom Filters
}

\author{
JONATHAN L. DAUTRICH JR., Google, USA \\ CHINYA V. RAVISHANKAR, University of California, Riverside, USA
}

\begin{abstract}
Current Bloom Filters tend to ignore Bayesian priors as well as a great deal of useful information they hold, compromising the accuracy of their responses. Incorrect responses cause users to incur penalties that are both application- and item-specific, but current Bloom Filters are typically tuned only for static penalties. Such shortcomings are problematic for all Bloom Filter variants, but especially so for Time-decaying Bloom Filters, in which the memory of older items decays over time, causing both false positives and false negatives.

We address these issues by introducing inferential filters, which integrate Bayesian priors and information latent in filters to make penalty-optimal, query-specific decisions. We also show how to properly infer insertion times in such filters. Our methods are general, but here we illustrate their application to inferential time-decaying filters to support novel query types and sliding window queries with dynamic error penalties.

We present inferential versions of the Timing Bloom Filter and Generalized Bloom Filter. Our experiments on real and synthetic datasets show that our methods reduce penalties for incorrect responses to slidingwindow queries in these filters by up to $70 \%$ when penalties are dynamic.
\end{abstract}

CCS Concepts: • Information systems $\rightarrow$ Probabilistic retrieval models; Stream management;

Additional Key Words and Phrases: Bloom filters, statistical analysis, bayesian priors, sliding windows, inferential bloom filters

\section{ACM Reference format:}

Jonathan L. Dautrich Jr. and Chinya V. Ravishankar. 2019. Inferring Insertion Times and Optimizing Error Penalties in Time-decaying Bloom Filters. ACM Trans. Database Syst. 44, 2, Article 7 (March 2019), 32 pages. https://doi.org/10.1145/3284552

\section{INTRODUCTION}

Bloom Filters are probabilistic data structures used for set membership queries [34]. Although this data structure derives its name from Reference [2], the method is equivalent to the Zatocoding technique described in 1951 by Mooers [25] for encoding information onto punched cards. Bloom Filters have been widely applied in areas where a concise but approximate representation of sets is required. They have, for example, been used to estimate join sizes and to speed up joins [18, 22,

This work was supported by Grants No. N00014-07-C-0311 from the Office of Naval Research, No. CPS-1330110 and No. IIS-1527984 from the National Science Foundation, and by the National Physical Science Consortium Graduate Fellowship. Authors' addresses: J. L. Dautrich Jr., Google, 19510 Jamboree Road, Irvine, CA, 92612, USA; email: jjldjr@gmail.com; C. V. Ravishankar, University of California, Riverside, 454 Winston Chung Hall, Riverside, CA, 92521, USA; email: ravi@ cs.ucr.edu.

Permission to make digital or hard copies of all or part of this work for personal or classroom use is granted without fee provided that copies are not made or distributed for profit or commercial advantage and that copies bear this notice and the full citation on the first page. Copyrights for components of this work owned by others than the author(s) must be honored. Abstracting with credit is permitted. To copy otherwise, or republish, to post on servers or to redistribute to lists, requires prior specific permission and/or a fee. Request permissions from permissions@acm.org.

(C) 2019 Copyright held by the owner/author(s). Publication rights licensed to ACM.

0362-5915/2019/03-ART7 \$15.00

https://doi.org/10.1145/3284552 
26, 27]. Oracle releases 10.2.0.x and later use Bloom Filters to reduce traffic between parallel query slaves during join processing. Bloom Filters have natural applications in stream processing and duplicate detection [24]. Other applications include maintaining differential files [12] and for spell checking $[10,23]$. For surveys of variant Bloom Filter designs and comparisons, see References [3, 20].

A Bloom Filter $\mathcal{F}$ comprises an array of $m$ cells and $k$ hash functions $h_{1}, \ldots, h_{k}$. An item $x$ is inserted into $\mathcal{F}$ by updating the contents of the cells at indices $h_{1}(x), \ldots, h_{k}(x)$. The contents of $\mathcal{F}$ 's cells define its state $\widehat{\mathcal{F}}$. The set of items inserted into $\mathcal{F}$ is denoted $\{\mathcal{F}\}$.

The Classical Bloom Filter [2] tests if an item $x \in\{\mathcal{F}\}$, returning Pos if $x \in\{\mathcal{F}\}$ and Neg if $x \notin\{\mathcal{F}\}$. Inserted items are never deleted, so $\mathcal{F}$ may become saturated, leading to false-positive errors, returning Pos even when $x \notin\{\mathcal{F}\}$.

A Time-decaying Bloom Filter [8, 15, 16, 38, 39], in contrast, supports queries that ask how recently $x$ was inserted. New insertions obscure information from older ones, so the memory of old items decays with time, limiting saturation even for continuous streams of item insertions.

Definition 1. The insertion age $I_{x}$ of item $x$ is a random variable denoting the number of items inserted since $x$ was last inserted. If $x$ was never inserted, then we define $I_{x}=\perp$. Different $I_{x}$ values represent mutually exclusive events.

Time-decaying filters answer retrospective queries, whose predicates reference insertion ages. A typical retrospective query is the sliding window query, which asks whether $x$ was one of the last $w$ items inserted $\left(I_{x}<w\right)$. Insertion history is only approximated by $\widehat{\mathcal{F}}$, so we may commit falsepositive errors, returning Pos when $I_{x} \geq w$, or false-negative errors, returning NeG when $I_{x}<w$. Such errors incur penalties ultimately borne by the application using $\mathcal{F}$.

Current time-decaying filters waste much of the useful information in $\widehat{\mathcal{F}}$. For example, in References $[8,33]$, cell counters are decremented at each insertion, and hence embed information about insertion age. Yet, these filters check only whether these counters are zero, discarding the more detailed information available. Even filters that do consider exact counts [15] do not provide a clear framework for using counter values.

More importantly, filters typically operate using "forward" probabilities, ignoring Bayesian priors. Our work is motivated by the observation that ignoring priors is fundamentally incorrect, and often leads to worse results than using no filter at all. A similar result was reported in Reference [31].

\subsection{Inferential Time-decaying Filters}

We present inferential time-decaying filters to address these issues. Inferential filters combine latent information in $\widehat{\mathcal{F}}$ with Bayesian priors to infer posterior probabilities.

Definition 2. $P\left(I_{x}=i \mid \widehat{\mathcal{F}}\right)$ is the posterior probability that item $x$ has insertion age $i$, given the filter state $\widehat{\mathcal{F}}$.

A standard time-decaying filter uses limited information from $\widehat{\mathcal{F}}$ to respond Pos or NEG to sliding window queries. An inferential time-decaying filter uses $P\left(I_{x}=i \mid \widehat{\mathcal{F}}\right)$ to achieve greater flexibility and accuracy in answering queries.

False positives/negatives incur application-dependent error penalties. Standard filters may be tuned to minimize static penalties that are fixed at filter design time. In reality, however, penalties vary by queried item, time, and context. A wrong decision on a high-value item costs more than one on a low-value item. Scenarios with query-specific penalties include duplicate detection for 
items with different values [1], distributed caches with item-specific access times [32], and web crawler caches when pages vary in importance [28].

Optimally, each membership decision should be made dynamically, query-by-query, and minimize expected penalty. Inferential time-decaying filters infer the sliding window posterior probability $P\left(I_{x}<w \mid \widehat{\mathcal{F}}\right)=\sum_{i=0}^{w-1} P\left(I_{x}=i \mid \widehat{\mathcal{F}}\right)$ for each sliding window query. They then use this posterior to compute expected penalties of Pos and NEG responses and make minimum-penalty decisions for each query.

Inferential filters also support novel retrospective queries, beyond enabling minimum-cost decisions. For instance, $P\left(I_{x}=i \mid \widehat{\mathcal{F}}\right)$ can be used to find the most likely insertion age for $x$. Aggregating over all $i$ gives the expected insertion age. As far as we know, our work is the first to support such queries using Bloom Filters.

\subsection{Contributions}

As noted above, filters have typically operated using "forward" probabilities, ignoring Bayesian priors. Ignoring priors, however, is fundamentally incorrect. We show how to turn existing standard filters into inferential ones, using Bayesian priors and latent information in $\widehat{\mathcal{F}}$. Section 2 outlines our inferential filter framework. We focus primarily on time-decaying filters, but our framework also immediately yields a more accurate version of the Classical Bloom Filter (see Section 2.4).

We show details of how to develop an inferential version of Timing Bloom Filters (TBF) [38], and use it for sliding window queries. We also develop standard and inferential versions of a spaceefficient TBF variant called the Block TBF (BTBF), conceptualized in Reference [38]. We discuss standard and inferential BTBFs in Sections 3 and 4, respectively.

We also develop an inferential version of the Generalized Bloom Filter (GBF) [16], in which each cell is a single bit. As new items are inserted, memory of old items steadily decays. The standard GBF has no built-in notion of a sliding window, so the GBF is less accurate than the BTBF when the window width $w$ is fixed, but the inferential GBF can support windows of different $w$ for each query. We discuss standard and inferential GBFs in Sections 5 and 5.3, respectively.

In Section 6, we compare the net penalty incurred by the standard and inferential BTBF and GBF on real and synthetic data streams, randomly varying error penalties for sliding window queries. We also compare them against two baselines, the first of which uses only on prior probabilities. The second is a simple buffer that stores hashes of items in the window. The buffer upper bounds the accuracy of sliding window techniques that require stored items, including those using Counting Bloom Filters $[13,35,36]$. Our results show that the inferential filters improve substantially upon the standard filters, reducing penalties when Bayesian priors are known. We discuss related work in Section 7.

\section{INFERENTIAL FILTER FRAMEWORK}

Bloom Filter variants commonly consist of an array of $m$ cells and $k$ independent hash functions $h_{1}, \ldots, h_{k}$, where hash $h_{i}$ maps an item $x$ to a cell $h_{i}(x)$ in the filter. Notation from Sections 1 and 2 is summarized in Table 1.

Definition 3. The set $R_{x}$ of cells touched by item $x$ is given by $R_{x}=\left\{h_{1}(x), \ldots, h_{k}(x)\right\}$.

To insert an item $x$ into filter $\mathcal{F}$, we update each cell in $R_{x}$ according to the rules of $\mathcal{F}$. To query for $x$, we inspect each cell in $R_{x}$ and return Pos or NEG as appropriate. Let $n$ be the number of past insertions. 
Table 1. General Notation

\begin{tabular}{|l|l|}
\hline $\mathcal{F}$ & A Bloom Filter \\
\hline$\widehat{\mathcal{F}}$ & The state of Bloom Filter $\mathcal{F}$ \\
\hline$\{\mathcal{F}\}$ & Set of all items inserted into a filter \\
\hline$x$ & Item to be inserted or queried \\
\hline$n=|\{\mathcal{F}\}|$ & Total number of items inserted \\
\hline$w$ & Width of sliding window \\
\hline$U$ & Universe of items inserted/queried \\
\hline$p_{x}$ & Sample probability of $x$ \\
\hline$m$ & Number of cells in filter \\
\hline$k$ & Number of hash functions used in filter \\
\hline$h, h_{1}(x)$ & Hash function, cell touched by $h_{1}$ on $x$ \\
\hline$I_{x}, i$ & Number of insertions since $x$ was last inserted \\
\hline$\perp$ & $I_{x}=\perp$ means $x$ was never inserted \\
\hline$R_{x}$ & Cells touched by $k$ hashes applied to $x$ \\
\hline$r_{x}$ & $\left|R_{x}\right|$ \\
\hline$c_{x}$ & For standard filter, number of 1-bits in $R_{x}$ \\
\hline$P_{F P}$ & False-positive probability of standard filter \\
\hline$P(i)$ & Prior prob. $i$ insertions since $x$ was last inserted \\
\hline$P\left(R_{x} \mid i\right)$ & Conditional probability of $R_{x}$ given $I_{x}=i$ \\
\hline$P\left(i \mid R_{x}\right)$ & $\begin{array}{l}\text { Posterior prob. of } I_{x}=i \text { insertions since } x \text { was last inserted, given contents } \\
\text { of cells in } R_{x}\end{array}$ \\
\hline$P\left(I_{x}<w \mid R_{x}\right)$ & Posterior prob. $x$ one of last $w$ insertions \\
\hline$D(j)$ & Expected num. distinct items in $j$ inserts \\
\hline
\end{tabular}

\subsection{The Classical Bloom Filter}

The Classical Bloom Filter [2] represents the set $\{\mathcal{F}\}$ of all items inserted into the filter $(n=|\{\mathcal{F}\}|)$. Each cell is a single bit initialized to 0 . To insert $x$, each cell in $R_{x}$ is set to 1 . Some cells may be touched by multiple items. A query for $x$ returns Pos if and only if all cells in $R_{x}$ are 1 .

Figure 1 shows inserts and possible query outcomes for a Classical Bloom filter. Cells are never reset to 0 , so all cells in $R_{x}$ remain 1 if $x \in\{\mathcal{F}\}$. There are no false negatives, but a false positive occurs if $x \notin\{\mathcal{F}\}$ but every cell in $R_{x}$ has been touched by some item, as for $x_{3}$.

Let $r_{x}=\left|R_{x}\right|$. The probability that a given cell is not touched by a given insertion is $(1-1 / \mathrm{m})^{k}$. Thus, the probability that a given cell is touched by at least one of the $n$ items in $\{\mathcal{F}\}$ is $(1-(1-$ $1 / m)^{k n}$ ). When $x \notin\{\mathcal{F}\}$, the false-positive probability that all $r_{x}$ cells in $R_{x}$ are set to 1 (touched) by at least one item in $\{\mathcal{F}\}$ is

$$
P_{F P} \approx\left(1-\left(1-\frac{1}{m}\right)^{k n}\right)^{r_{x}}
$$

\subsection{Analytical Approximations}

$P_{F P}$ in Equation (1) is often approximated by replacing $r_{x}$ with $k$, as collisions are rare if $m \gg k$. Equation (1) assumes that cell touches in $R_{x}$ independent events, which is strictly incorrect [6]. Such approximations usually have little impact on accuracy [6] but greatly simplify analysis. We make similar assumptions in our article when computing posteriors. Our experiments show that the posteriors are generally accurate enough to substantially reduce error penalties. 


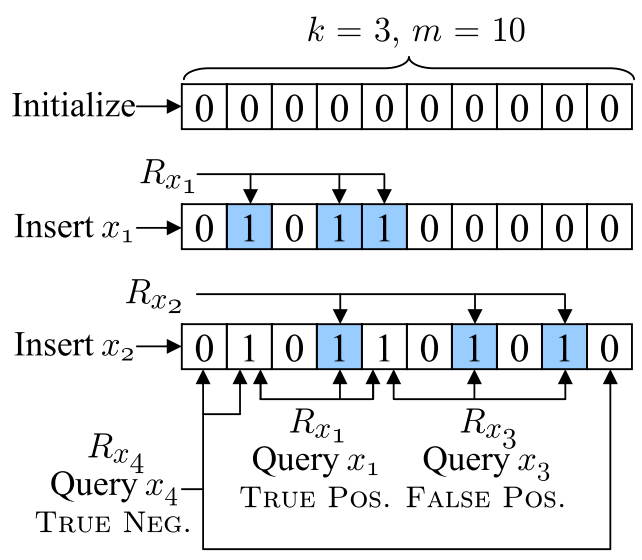

Fig. 1. Inserts and queries on a Classical Bloom Filter holding $\left\{x_{1}, x_{2}\right\}$.

\subsection{Probability Functions}

The posterior $P\left(I_{x}=i \mid \widehat{\mathcal{F}}\right)$ is conditioned on $\widehat{\mathcal{F}}$, the filter state, which includes all cells in $\mathcal{F}$. However, most information relevant to $x$ is present in the cells $R_{x}$.

Definition 4. $P\left(I_{x}=i \mid R_{x}\right)$, also denoted $P\left(i \mid R_{x}\right)$, is the posterior probability that exactly $i$ insertions occurred since $x$ was last inserted, given the current contents of cells $R_{x}$.

To turn a standard filter into an inferential one, we must compute $P\left(i \mid R_{x}\right)$, based on the filter's contents and on the prior probability that $I_{x}=i$. From Bayes' theorem,

$$
P\left(i \mid R_{x}\right)=\frac{P(i) P\left(R_{x} \mid i\right)}{P\left(R_{x}\right)}, \quad \text { where }
$$

$P\left(I_{x}=i\right)$ or $P(i)$ is the prior probability that exactly $i$ insertions occurred since $x$ was last inserted, $P\left(R_{x}\right)$ is the prior probability that cells $R_{x}$ have their current contents, and $P\left(R_{x} \mid I_{x}=i\right)$ or $P\left(R_{x} \mid i\right)$ is the conditional probability that cells $R_{x}$ have their current contents, given that exactly $i$ insertions occurred since $x$ was last inserted.

\subsubsection{Computing Prior Probability $P(i)$.}

Definition 5. The sample probability mass function $p_{x}$ is the probability that $x$ is the next item to be inserted.

Let $U$ be the universe of all items that may be inserted or queried. For any two items $x \neq y, p_{x}$ and $p_{y}$ may differ, but we assume that $p_{x}$ itself is time-invariant, giving

$$
P(i)=\left\{\begin{array}{ll}
p_{x}\left(1-p_{x}\right)^{i} & \text { if } i \neq \perp(0 \leq i<n) \\
\left(1-p_{x}\right)^{n} & \text { if } i=\perp
\end{array} .\right.
$$

$P(\perp)$ is the probability that $x$ was not inserted thus far, and $P(i), i \neq \perp$ is the probability that $x$ was inserted, followed by $i$ items other than $x$. We say the data stream is continuous when the number of past insertions $n$ goes to infinity, giving

$$
\lim _{n \rightarrow \infty} P(i)=\left\{\begin{array}{ll}
p_{x}\left(1-p_{x}\right)^{i} & \text { if } i \neq \perp \\
0 & \text { if } i=\perp
\end{array} .\right.
$$


Often, we need $P\left(\alpha \leq I_{x}<\beta\right)$ for $0 \leq \alpha<\beta$ :

$$
\begin{aligned}
P\left(\alpha \leq I_{x}<\beta\right) & =\sum_{i=\alpha}^{\beta-1} P(i)=p_{x} \sum_{i=\alpha}^{\beta-1}\left(1-p_{x}\right)^{i} \\
& =\left(1-p_{x}\right)^{\alpha}-\left(1-p_{x}\right)^{\beta} \\
& =\left(1-p_{x}\right)^{\alpha}\left(1-\left(1-p_{x}\right)^{\beta-\alpha}\right) .
\end{aligned}
$$

2.3.2 Computing Posterior $P\left(i \mid R_{x}\right) . P\left(R_{x}\right)$ in Equation (2) can be written as a marginal sum over $P(i) P\left(R_{x} \mid i\right)$, giving

$$
P\left(i \mid R_{x}\right)=\frac{P(i) P\left(R_{x} \mid i\right)}{P(\perp) P\left(R_{x} \mid \perp\right)+\sum_{i^{\prime}=0}^{n-1} P\left(i^{\prime}\right) P\left(R_{x} \mid i^{\prime}\right)} .
$$

We still need $P\left(R_{x} \mid i\right)$ and $\sum_{i^{\prime}=0}^{n-1} P\left(i^{\prime}\right) P\left(R_{x} \mid i^{\prime}\right)$. Both challenges are filter-specific, so we address them for the BTBF and GBF in Sections 4 and 5.3, respectively.

2.3.3 Retrospective Queries. Inferential time-decaying filters use $P\left(i \mid R_{x}\right)$ in responding to retrospective queries. $\max _{i} P\left(i \mid R_{x}\right)$ gives the highest probability choice $i$ for $I_{x}$, which is when $x$ was most likely last inserted.

For a continuous stream $(n \rightarrow \infty)$, we get

$$
\lim _{n \rightarrow \infty} P\left(i \mid R_{x}\right)=\frac{P(i) P\left(R_{x} \mid i\right)}{\sum_{i^{\prime}=0}^{\infty} P\left(i^{\prime}\right) P\left(R_{x} \mid i^{\prime}\right)} .
$$

The expected number of insertions after $x$ 's last insertion is

$$
E\left[I_{x} \mid R_{x}\right]=\lim _{n \rightarrow \infty} \sum_{i=0}^{n} i \cdot P\left(i \mid R_{x}\right) .
$$

We can also derive the sliding window posterior

$$
\begin{aligned}
\lim _{n \rightarrow \infty} P\left(I_{x}<w \mid R_{x}\right)=\sum_{i=0}^{w-1} \lim _{n \rightarrow \infty} P\left(i \mid R_{x}\right) \\
=\sum_{i=0}^{w-1} \frac{P(i) P\left(R_{x} \mid i\right)}{\sum_{i^{\prime}=0}^{\infty} P\left(i^{\prime}\right) P\left(R_{x} \mid i^{\prime}\right)}=\frac{\sum_{i=0}^{w-1} P(i) P\left(R_{x} \mid i\right)}{\sum_{i=0}^{\infty} P(i) P\left(R_{x} \mid i\right)} \\
=1-\frac{\sum_{i=w}^{\infty} P(i) P\left(R_{x} \mid i\right)}{\sum_{i=0}^{\infty} P(i) P\left(R_{x} \mid i\right)} .
\end{aligned}
$$

\subsection{Example: Classical Bloom Filters}

As warm-up, we develop an inferential version of Classical Bloom filters (Section 2.1), by computing the posterior $P\left(x \in\{\mathcal{F}\} \mid R_{x}\right)$. Since this filter is not time-decaying, we do not need the full power of our approach, but we show our results to be consistent with the simpler derivation in Reference [31]. We also show how to obtain optimal responses from the inferential Classical Bloom Filter, given item-specific prior probabilities $p_{x}$ and query-specific error penalties. Since $n=|\{\mathcal{F}\}|$,

$$
\begin{aligned}
P\left(x \in\{\mathcal{F}\} \mid R_{x}\right) & =P\left(I_{x}<n \mid R_{x}\right)=\sum_{i=0}^{n-1} P\left(i \mid R_{x}\right) \\
& =\frac{\sum_{i=0}^{n-1} P(i) P\left(R_{x} \mid i\right)}{P(\perp) P\left(R_{x} \mid \perp\right)+\sum_{i=0}^{n-1} P(i) P\left(R_{x} \mid i\right)} .
\end{aligned}
$$


Let $r_{x}=\left|R_{x}\right|$. Let $c_{x}$ cells (bits) in $R_{x}$ be set to 1 ,

$$
P\left(R_{x} \mid i\right)=\left\{\begin{array}{l}
1 \quad \text { if } c_{x}=r_{x} \text { and } 0 \leq i<n \\
0 \quad \text { if } c_{x} \neq r_{x} \text { and } 0 \leq i<n \\
\left(1-\left(1-\frac{1}{m}\right)^{k n}\right)^{c_{x}}\left(\left(1-\frac{1}{m}\right)^{k n}\right)^{r_{x}-c_{x}} \text { if } i=\perp
\end{array} .\right.
$$

If $x$ was inserted $(0 \leq i<n)$, then all cells in $R_{x}$ must be $1\left(c_{x}=r_{x}\right)$. If $x$ was not inserted $(i=\perp)$, then every one of the $c_{x}$ 1-cells in $R_{x}$ must have been touched (set) by some combination of the $n$ insertions, while the remaining $r_{x}-c_{x} 0$-cells in $R_{x}$ must not have been touched by any insertion.

Theorem 1. The posterior probability that $x$ was inserted into the Classical Bloom Filter is given by

$$
P\left(I_{x}<n \mid R_{x}\right)= \begin{cases}0 & \text { if } c_{x} \neq r_{x} \\ \frac{1}{1+\frac{\left(1-p_{x}\right)^{n} P_{F P}}{1-\left(1-p_{x}\right)^{n}}} & \text { if } c_{x}=r_{x}\end{cases}
$$

where $P_{F P}$ is as in Equation (1).

Proof. $P\left(I_{x}<n \mid R_{x}\right)$ is given by Equation (11), $P(i)$ by Equation (3), and $P\left(R_{x} \mid i\right)$ by Equation (12).

CASE $c_{x} \neq r_{x}$ :

$$
P\left(I_{x}<n \mid R_{x}\right)=\frac{\sum_{i=0}^{n-1} P(i) \cdot 0}{P(\perp) P\left(R_{x} \mid \perp\right)+\sum_{i=0}^{n-1} P(i) \cdot 0}=0 .
$$

CASE $c_{x}=r_{x}$ :

$$
\begin{aligned}
& P\left(I_{x}<n \mid R_{x}\right)= \\
& \frac{\sum_{i=0}^{n-1} P(i) \cdot 1}{P(\perp)\left(1-\left(1-\frac{1}{m}\right)^{k n}\right)^{r_{x}}+\sum_{i=0}^{n-1} P(i) \cdot 1} \\
& =\frac{(1-P(\perp))}{P(\perp) \cdot P_{F P}+(1-P(\perp))} \\
& =\frac{1}{1+\frac{P(\perp) \cdot P_{F P}}{1-P(\perp)}}=\frac{1}{1+\frac{\left(1-p_{x}\right)^{n} \cdot P_{F P}}{1-\left(1-p_{x}\right)^{n}}} .
\end{aligned}
$$

For the Classical Bloom Filter it is common to assume that $r_{x}=k, k \approx(m / n) \ln 2$ and that $P_{F P} \approx$ $\left(1-e^{-k n / m}\right)^{k}$, as in References [16,31,33,38]. Doing so gives $P_{F P} \approx(1 / 2)^{(m / n) \ln 2}$, so when $c_{x}=r_{x}$, rearranging Theorem 1 and substituting $P\left(I_{x}<n\right)=1-\left(1-p_{x}\right)^{n}$ gives

$$
P\left(I_{x}<n \mid R_{x}\right) \approx \frac{P\left(I_{x}<n\right)}{P(\perp)\left(\frac{1}{2}\right)^{(m / n) \ln 2}+P\left(I_{x}<n\right)},
$$

which is consistent with the probability expressions in Reference [31].

\subsection{Expected Number of Distinct Items}

The accuracy of our posteriors can be improved if we know the expected number of distinct items inserted during $j$ insertions, which we label $D(j) . D(j)$ depends on the distribution of $p_{x}$ for $x \in U$. 
Applying linearity of expectation, we can find $D(j)$ by summing, over all $x \in U$, the probability that $x$ is inserted at least once, given by

$$
D(j)=\sum_{x \in U}\left(1-\left(1-p_{x}\right)^{j}\right)
$$

When items are sampled from the uniform distribution, we have $p_{x}=1 /|U|$ for all $x \in U$, and Equation (15) becomes

$$
D(j)=|U|\left(1-\left(1-\frac{1}{|U|}\right)^{j}\right) .
$$

If the item probabilities follow a Zipf-like discrete power law $p_{x}=1 /\left(H_{|U|} \cdot x\right)$, then it is shown in Reference [37] that

$$
D(j) \approx \frac{j}{H_{|U|}}\left(1-\gamma+\ln \frac{|U| \cdot H_{|U|}}{j}\right),
$$

where $H_{|U|}=\sum_{i=1}^{|U|} 1 / i$ is the $|U|$ th harmonic number and $\gamma=0.57721566 \ldots$ is Euler's constant.

When real-world distributions are hard to model analytically, we can experimentally determine $D(j)$ for some $j$ values, and interpolate intermediate values. Our experience suggests that piecewise logarithmic interpolation generally yields acceptable results. If we know $D\left(j_{1}\right)$ and $D\left(j_{3}\right)$, then we can interpolate $D\left(j_{2}\right)$ for $j_{1}<j_{2}<j_{3}$ as follows:

$$
\ln D\left(j_{2}\right)=\ln D\left(j_{1}\right)+\frac{\left(\ln \frac{D\left(j_{3}\right)}{D\left(j_{1}\right)}\right)\left(\ln \frac{j_{2}}{j_{1}}\right)}{\left(\ln \frac{j_{3}}{j_{1}}\right)} .
$$

\subsection{Minimum-Penalty Decisions}

As noted in Section 1, penalties for incorrect responses may be query-specific, but a standard filter can only be tuned to fixed false-positive/false-negative rates. Inferential filters use posteriors to make better-informed, query-specific decisions.

For sliding window queries, inferential filters return the sliding window posterior $P\left(I_{x}<w \mid R_{x}\right)$. Let $\$_{F P}$ and $\$_{F N}$ be the penalties for false-positive/false-negative errors, respectively. Correct responses incur no penalty. The expected penalty of Pos is $E_{\mathrm{Pos}}=\$_{F P} \cdot\left(1-P\left(I_{x}<w \mid R_{x}\right)\right)$, and of Neg is $E_{\mathrm{NEG}}=\$_{F N} \cdot P\left(I_{x}<w \mid R_{x}\right)$. We compute both and return Pos if $E_{\mathrm{Pos}} \leq E_{\mathrm{NEG}}$, and NEG otherwise.

\section{STANDARD TIMING BLOOM FILTERS}

The Timing Bloom Filter (TBF) [38] is designed to answer sliding window queries. Here, we describe the standard TBF and its extension, the standard Block Timing Bloom Filter (BTBF). We will present the inferential BTBF in Section 4. Table 2 summarizes the relevant notation.

\subsection{Timing Bloom Filters}

The TBF consists of $k$ hash functions and an array of $m$ cells, each of which is a timer with $b p t$ bits. Each timer $\theta$ holds a timestamp $\theta . T \in\left\{0, \ldots, T_{\Omega}\right\} \cup\left\{T_{\varepsilon}\right\}$, where $T_{\varepsilon}$ denotes an expired timestamp, defined below. The filter maintains a single current timestamp $T_{+}$, where $T_{+}$cycles through the range $\left[0, T_{\Omega}\right]$ as items are inserted. 
Table 2. TBF/BTBF Notation

\begin{tabular}{|l|l|}
\hline$b p t$ & Number of bits per timer \\
\hline$\theta, \theta . T$ & Timer, timestamp stored by timer \\
\hline$T_{+}$ & Current timestamp \\
\hline$T_{\Omega}$ & Maximum timestamp value \\
\hline$T_{\varepsilon}$ & Expired timestamp value \\
\hline$T_{x}$ & Oldest timestamp in $R_{x}$ \\
\hline$\lambda(T)$ & Age of timestamp $T$ \\
\hline$\lambda_{x}$ & Age of oldest timestamp: $\lambda_{x}=\lambda\left(T_{x}\right)$ \\
\hline $\mathcal{P}$ & Padding size \\
\hline$B$ & Insertion block size \\
\hline$b$ & Insertions since last $T_{+}$increment \\
\hline$C_{x}$ & Timers in $R_{x}$ with oldest timestamp $T_{x}$ \\
\hline$c_{x}$ & $\left|C_{x}\right|$ \\
\hline$F\left(c_{x}, r_{x}, j\right)$ & $\begin{array}{l}\text { Prob. specific } c_{x} \text { of } r_{x} \text { timers untouched during } j \text { inserts, other } r_{x}-c_{x} \\
\text { touched }\end{array}$ \\
\hline$F(\cdot)$ & Short for $F\left(r_{x}, c_{x},\left(\lambda_{x}-1\right) B+b\right)$ \\
\hline$G\left(r_{x}, c_{x}, \lambda_{x}\right)$ & $\begin{array}{l}\text { Prob. } c_{x} \text { timers touched by } B \text { inserts, same } c_{x} \text { timers not touched by any of } \\
\text { subsequent }\left(\lambda_{x}-1\right) B+b \text { inserts, other } r_{x}-c_{x} \text { touched }\end{array}$ \\
\hline$G(\cdot)$ & Short for $G\left(r_{x}, c_{x}, \lambda_{x}\right)$ \\
\hline
\end{tabular}

\subsubsection{TBF: Insert. We insert item $x$ into a TBF as follows:}

(1) For each timer $\theta \in R_{x}$, set $\theta . T \leftarrow T_{+}$.

(2) Increment $T_{+}: T_{+} \leftarrow\left(T_{+}+1\right) \bmod \left(T_{\Omega}+1\right)$.

Definition 6. The age $\lambda(\theta . T)$ of timestamp $\theta . T$ is defined as the number of times that $T_{+}$was incremented since the last time that we set $\theta . T$ to $T_{+}$.

When $\lambda(\theta . T) \geq w+1$, we say that $\theta . T$ has expired, and we set $\theta . T$ to the expired timestamp value $T_{\varepsilon}$. Thus, as soon as a timestamp $\theta . T$ is set to $T_{+}$, it has age $\lambda(\theta . T)=0$, but since increments occur immediately after insertions, $\lambda(\theta . T) \geq 1$ before queries arrive. We define $\lambda\left(T_{\varepsilon}\right)=\infty$.

3.1.2 TBF: Query. When we query the TBF for item $x$, it should return Pos whenever $I_{x}<w$, and Neg otherwise. To query, we examine each timer $\theta$ in $R_{x}$ and compute the age of its timestamp $\lambda(\theta . T)$. The TBF returns Neg if any $\theta \in R_{x}$ has an expired timestamp, and returns Pos otherwise, yielding false positives but no false negatives.

False Negatives: Since all $\theta \in R_{x}$ are set to $T_{+}$when $x$ is inserted, we know that $I_{x} \geq \lambda(\theta . T)-1$, for all $\theta \in R_{x}$. Therefore, if for any $\theta \in R_{x}, \theta$.T has expired, we know that $I_{x} \geq \lambda(\theta \cdot T)-1 \geq w$. Since we only return NEG when one of the $\theta$.T has expired, the TBF has no false negatives.

False Positives: A false-positive error occurs when no timestamp in $R_{x}$ has expired, but $I_{x} \geq$ $w$. The standard TBF only has false positives if all timers in $R_{x}$ were touched during the last $w$ insertions, none of which inserted $x$.

3.1.3 TBF: Marking Expired Timestamps. If any timestamp $\theta . T$ expires, then we must mark it expired $\left(\theta . T \leftarrow T_{\varepsilon}\right)$ before $T_{+}=\theta . T$ again. If we do not, then $\lambda(\theta . T)$ will cycle back to 0 , and we 


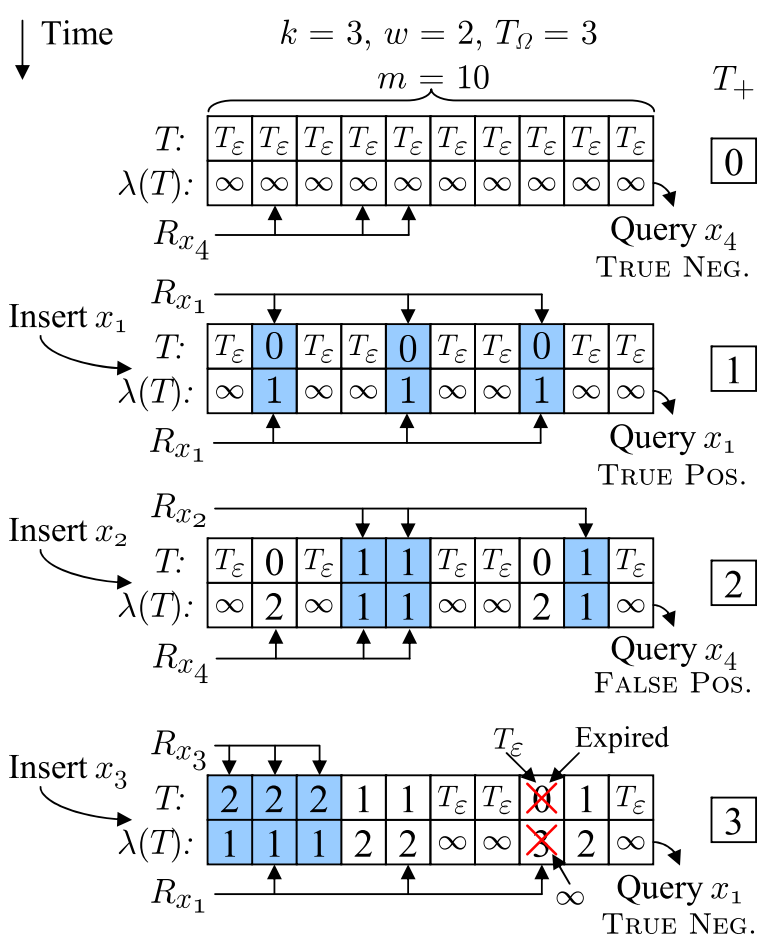

Fig. 2. Timing Bloom Filter inserts and queries. Timestamps touched by each insertion highlighted. Ages are relative to the updated $T_{+}$.

will not know that $\theta . T$ ought to be expired. ${ }^{1}$ If $T_{\Omega}=w$, then there are $w+1$ values for $T_{+}$, so it can only be incremented $w$ times without returning it to its current value. Thus, $w$ is the maximum timestamp age, and timestamps never get a chance to expire. Hence, to correctly support a window of width $w$, we must have $T_{\Omega} \geq w+1$. An example of a TBF with $T_{\Omega}=w+1$ is given in Figure 2 .

If we have the minimum $T_{\Omega}=w+1$, then once any timestamp $\theta$.T expires, we must set $\theta . T \leftarrow T_{\varepsilon}$ before the next insertion, which would set $T_{+} \leftarrow \theta$.T. Thus, to find all newly expired timestamps, we must check all $m$ timers after every insertion, which is too expensive. The solution in Reference [38] is to increase $T_{\Omega}$ by an amount we call padding.

Definition 7. The padding $\mathcal{P}$ is the difference between the chosen and minimum values for $T_{\Omega}$.

For a standard TBF, $\mathcal{P}=T_{\Omega}-w$. If $\mathcal{P}=1$, then $T_{\Omega}=w+2$, and we can recognize an expired timestamp up to one insertion after it first expires. Thus, we can split up the search for expired timestamps, such that we need only check half of the timers after each insertion. In general, with padding $\mathcal{P}$, we need only check $m /(\mathcal{P}+1)$ timers after each insertion. The use of padding is demonstrated in Figure 3.

A good rule of thumb is to set $\mathcal{P} \approx m / k$, so we need only check $O(k)$ timers per insertion. Since we already perform $O(k)$ hashes for each insertion, checking $O(k)$ timers is acceptable. As long as $m \approx w$, as is often the case, this choice of $\mathcal{P}$ increases $T_{\Omega}$ by less than $w$, so we need at most one extra bit per timer to accommodate the larger $T_{\Omega}$.

\footnotetext{
${ }^{1}$ If we assume that timers should never have age 0 , then we can actually let $T_{+}$cycle back to $\theta . T$, but not beyond, and treat its apparent age 0 as age $T_{\Omega}+1$. We can thus reduce the minimum $T_{\Omega}$ value by 1 , but we do not do so, since this assumption does not hold for Block Timing Bloom Filters.
} 


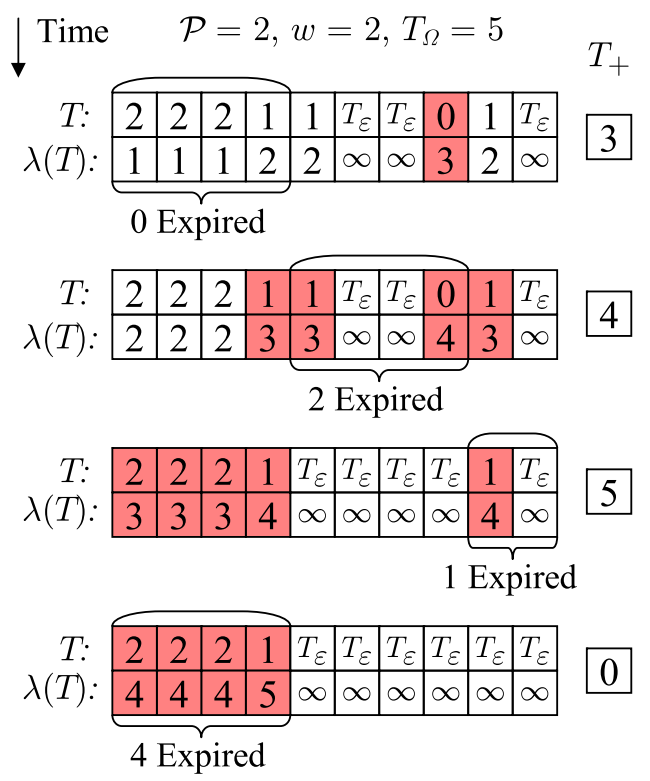

Fig. 3. Padding $\mathcal{P}$ in a Timing Bloom Filter, new insertions omitted for clarity. Newly expired timestamps (highlighted) can remain expired for $\mathcal{P}+1$ insertions before $T_{+}$cycles, so we need only check 4 timers/insertion.

\subsection{Block Timing Bloom Filters}

TBF has the problem that the $T_{\Omega}+2$ possible timestamps require it to use $O(\log w)$ bits per timer $(b p t)$. For example, the sample TBF in Reference [38] has a window size of $w=2^{20}$, requiring $21 \mathrm{bpt}$, including 1 bit for padding. It has $m=15,112,980$ timers, for a total of $21 \mathrm{~m}$ bits, and $21 \mathrm{~m} / w \approx 303$ bits/item in the window of interest, which is excessive. Given 303 bits/item, we could just hash all items in the window into a table using unique hashes. This setup matches TBF performance, and is simpler and more accurate.

We can reduce $b p t$ by incrementing $T_{+}$only after every block of $B>1$ insertions, where $B$ is the insertion block size. Using a larger $B$ reduces $b p t$, but uses fewer blocks to cover the window, resulting in a coarser approximation and more false positives (see Section 3.2.2). We call this scheme a Block Timing Bloom Filter (BTBF), due to its similarities to the Block Decaying Bloom Filter in Reference [33]. The BTBF was alluded to, but not developed, in Reference [38].

3.2.1 BTBF: Insert. Insertions into the BTBF proceed as for the TBF, except that we only increment $T_{+}$once for each block of $B$ insertions. A counter $b$ records the number of insertions since the last time $T_{+}$was incremented. If $B=1$, as in the standard TBF, then we always have $b=0$. After each insertion, if $b=B-1$, we increment $T_{+}$and set $b=0$. If $b<B-1$, then we increment $b$ and leave $T_{+}$unchanged.

Definition 6 for $\lambda(\theta . T)$ still holds, but our definition of an expired timestamp becomes more general:

Definition 8. In a BTBF, timestamp $\theta$.T has expired once its age $\lambda(\theta . T) \geq\left\lceil\frac{w-b}{B}\right\rceil+1$.

3.2.2 BTBF: Query. Like the TBF, the BTBF returns NEG if and only if some timestamp in $R_{x}$ has expired. Thus, it has false positives but no false negatives. 


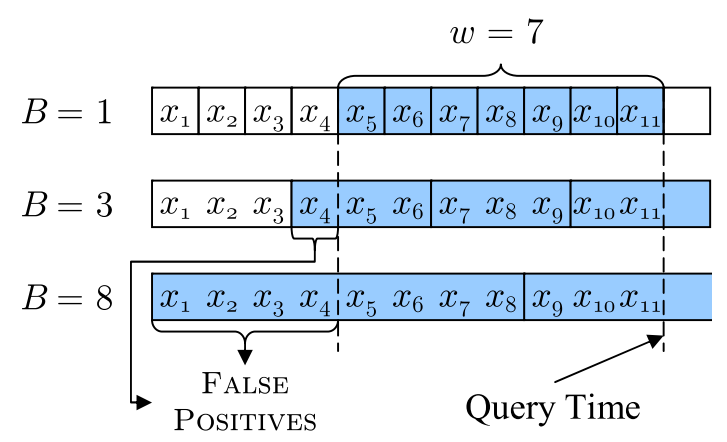

Fig. 4. For the BTBF to treat $x_{5}$ as in the window, it must treat all items in $x_{5}$ 's block as in the window. Larger blocks yield more false positives.

False Negatives: If $\lambda(x)=1$, then we know $I_{x} \geq b$. If $\lambda(x)=2$, then we know that $I_{x} \geq B+b$. In general, we have

$$
I_{x} \geq \begin{cases}(\lambda(\theta . T)-1) B+b & \text { if } \lambda(\theta . T)>0 \\ 0 & \text { if } \lambda(\theta . T)=0\end{cases}
$$

Therefore, if for any $\theta \in R_{x}, \theta . T$ has expired, we know that

$$
\begin{aligned}
I_{x} \geq(\lambda(\theta . T)-1) B+b & =\left(\left[\frac{w-b}{B}\right]+1-1\right) B+b \\
& \geq\left(\frac{w-b}{B}\right) B+b=w .
\end{aligned}
$$

That is, if any timestamp in $R_{x}$ has expired, $x$ is not in the window. NeG is returned only if at least one timestamp in $R_{x}$ has expired, so the BTBF has no false negatives.

False Positives: In a BTBF, false positives can occur in two ways. As for standard TBFs, they can occur if all timers in $R_{x}$ are touched by other recent inserts. False positives also occur if $x$ is one of the first items in a block, but only the latter items in the block are in the window. Such false positives are described below and illustrated in Figure 4.

Let $B>1$, and let $x_{1}$ and $x_{B}$ be the first and last items inserted during a given insertion block. If $I_{x_{B}}=w-1$, then $I_{x_{1}}=w+B-2$. Since the filter has no false negatives, a query for $x_{B}$ returns Pos. However, since $x_{1}$ and $x_{B}$ are part of the same insertion block, they use the same timestamp and are indistinguishable to the filter, so a query for $x_{1}$ must also return Pos. Since $I_{x_{1}} \geq w$, the response is a false positive. At any point, queries for an average of $B / 2$ items yield such false positives, so a larger $B$ gives a coarser sliding window approximation with more false positives.

3.2.3 BTBF: Marking Expired Timestamps. Marking expired timestamps and the use of padding are the same for the BTBF as for the TBF. However, the minimum $T_{\Omega}$ value is lower for BTBFs, allowing us to reduce $b p t$. To support a window of width $w$, we now need $T_{\Omega} \geq\left\lceil\frac{w}{B}\right\rceil+1$. We also now need only check $m /(B(\mathcal{P}+1))$ timers after each insertion, so we can choose $\mathcal{P} \approx m /(k B)$. An example of a BTBF with $\mathcal{P}=0, B=3, w=6$ is given in Figure 5.

\section{INFERENTIAL BTBF}

We now develop the inferential BTBF, which returns the sliding window posterior $\lim _{n \rightarrow \infty} P\left(I_{x}<\right.$ $\left.w \mid R_{x}\right)$, instead of just a binary Pos or NEG, in response to queries. We derive $\lim _{n \rightarrow \infty} P\left(I_{x}<w \mid R_{x}\right)$ directly using Equations (9) and (10). For the sake of brevity, we omit the limit notation in the rest of the article. 


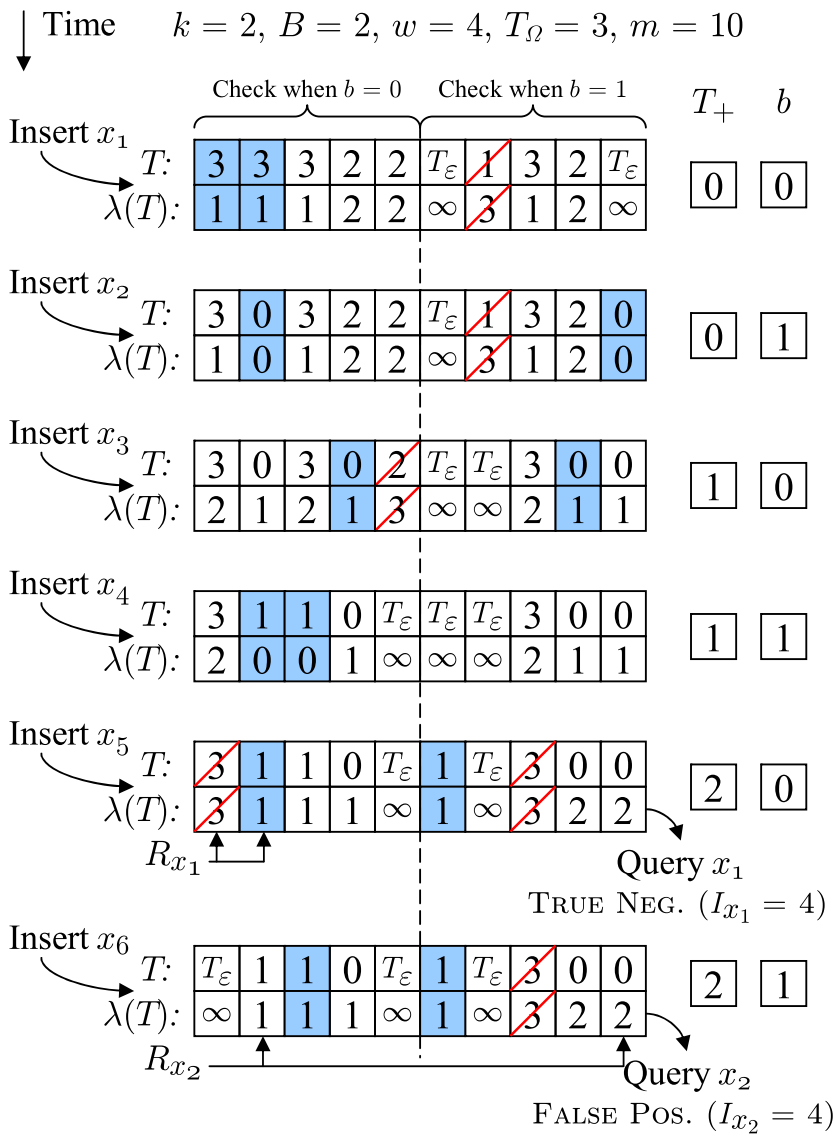

Fig. 5. A BTBF with no padding. Timers touched by the last insertion are highlighted. Timestamp $T$ expires once $\lambda(T) \geq 3$ and is shown with a slash until it is changed to $T_{\varepsilon}$. Since $B=2$, we need only check half the timers for expiration after each insertion.

Definition 9. $T_{x}$, the oldest timestamp in $R_{x}$ has age

$$
\lambda_{x}=\operatorname{MAx}\left\{\lambda(\theta . T) \mid \theta \in R_{x}\right\} .
$$

If any timestamp in $R_{x}$ has expired, then $\lambda_{x}=\infty$.

If $x$ had been inserted since the last time $T_{+}=T_{x}$, then all timers in $R_{x}$ would have been set to a more recent timestamp. Thus, if any of the timers in $R_{x}$ still have the timestamp they were given the last time $x$ was inserted, then it is only those timers with timestamp $T_{x}$ and age $\lambda_{x}$.

Definition 10. Let $C_{x}$ be the subset of timers in $R_{x}$ that have timestamps with age $\lambda_{x}$. That is,

$$
C_{x}=\left\{\theta \mid \theta \in R_{x} \wedge \lambda(\theta \cdot T)=\lambda_{x}\right\} .
$$

Let $r_{x}=\left|R_{x}\right|$ and $c_{x}=\left|C_{x}\right|$. The timers in $R_{x} \backslash C_{x}$ must have timestamps set by items other than $x$, so only the timers in $C_{x}$ could have been last touched by $x$, so that only timers in $C_{x}$ provide worthwhile information about when $x$ was last inserted $\left(I_{x}\right)$. Since all $c_{x}$ timers in $C_{x}$ have the same timestamp, with age $\lambda_{x}$, we can accurately compute posteriors given only $r_{x}, c_{x}$, and $\lambda_{x}$. That is, when we refer to $P\left(R_{x} \mid i\right)$, we are interested in the probability that $r_{x}, c_{x}$, and $\lambda_{x}$ have the values we observe, given that $I_{x}=i$. 


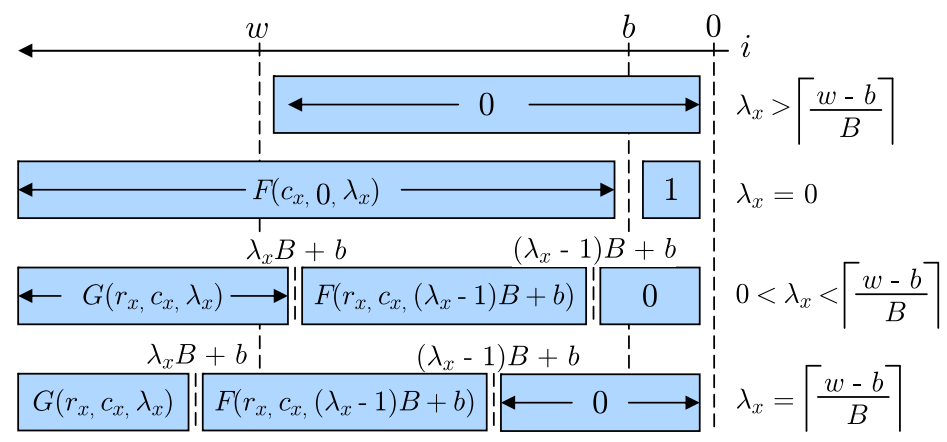

Fig. 6. $P\left(R_{x} \mid i\right)$ for different values of $\lambda_{x}$ and $i$.

The prior $P(i)$ is given by Equation (4). To get posteriors, we must sum over the conditional probability $P\left(R_{x} \mid i\right)$, which varies depending on the relationship between $i$ and $\lambda_{x}$, so we must handle different ranges of $\lambda_{x}$ separately. Figure 6 shows the different expressions for $P\left(R_{x} \mid i\right)$ derived below for each $\lambda_{x}$ case. We need the following function.

Definition 11. Let $F\left(r_{x}, c_{x}, j\right)$ be the probability that a specific subset of $c_{x}$ out of $r_{x}$ timers are not touched during $j$ insertions, and that the remaining $r_{x}-c_{x}$ timers are touched during the $j$ insertions. We approximate it as

$$
F\left(r_{x}, c_{x}, j\right) \approx\left(\left(1-\frac{1}{m}\right)^{k D(j)}\right)^{c_{x}}\left(1-\left(1-\frac{1}{m}\right)^{k D(j)}\right)^{r_{x}-c_{x}} .
$$

The probability that a given timer is not touched during a given insertion is $(1-1 / m)^{k}$. If we take $(1-1 / m)^{k j}$ to be the probability that a timer is not touched during $j$ insertions, then we ignore dependencies that arise when the same item is inserted more than once. We account for such dependencies by replacing $j$ with $D(j)$, where $D(j)$ gives the expected number of distinct items among $j$ insertions (see Section 2.5). Raising a probability to an expectation is not entirely valid, but it is an efficient and adequate approximation here, as our approximation error results show (Section 6.1.4).

\subsection{Case $\lambda_{x}>\left\lceil\frac{w-b}{B}\right\rceil$}

THeorem 2. If $\lambda_{x}>\left\lceil\frac{w-b}{B}\right\rceil$, then $P\left(I_{x}<w \mid R_{x}\right)=0$.

Proof. If $\lambda_{x}>\left\lceil\frac{w-b}{B}\right\rceil$, then at least one timestamp in $R_{x}$ has expired, so we know for certain that $I_{x} \geq w$, and thus $P\left(R_{x} \mid i\right)=0$ for $0 \leq i<w$, giving

$$
\begin{aligned}
P\left(I_{x}<w \mid R_{x}\right) & =\frac{\sum_{i=0}^{w-1} P(i) P\left(R_{x} \mid i\right)}{\sum_{i=0}^{\infty} P(i) P\left(R_{x} \mid i\right)}=\frac{\sum_{i=0}^{w-1} P(i) \cdot 0}{\sum_{i=0}^{\infty} P(i) P\left(R_{x} \mid i\right)} \\
& =0 .
\end{aligned}
$$

$P\left(I_{x}<w \mid R_{x}\right)=0$ when timestamps in $R_{x}$ have expired, because the standard BTBF has no false negatives.

\subsection{Case $\lambda_{x}=0$}

LEMMA 4.1. If $\lambda_{x}=0$, then

$$
P\left(R_{x} \mid i\right)=\left\{\begin{array}{ll}
1 & \text { if } i<b \\
F\left(r_{x}, 0, b\right) & \text { if } i \geq b
\end{array} .\right.
$$


Proof. If $\lambda_{x}=0$, then all timers in $R_{x}$ must have timestamp $T_{+}$with age 0 , so $c_{x}=r_{x}$.

CASE $i<b$ : If $i<b$, then $x$ would have been inserted since $T_{+}$was last incremented, and all timers in $R_{x}$ must have had their timestamps set to $T_{+}$and could not have been changed since, so $P\left(R_{x} \mid i\right)=1$.

CASE $i \geq b$ : If $i \geq b$, then $x$ would have been most recently inserted before $T_{+}$was last incremented. Thus, for all the timers in $R_{x}$ to have timestamp $T_{+}$, every one of the $r_{x}$ timers must have been touched through some combination of the last $b$ items inserted, none of which were $x$. The probability that this event occurs is $F\left(r_{x}, 0, b\right)$.

Theorem 3. If $\lambda_{x}=0$, then

$$
P\left(I_{x}<w \mid R_{x}\right)=1-\frac{\left(1-p_{x}\right)^{w}}{\frac{1-\left(1-p_{x}\right)^{b}}{F\left(r_{x}, 0, b\right)}+\left(1-p_{x}\right)^{b}} .
$$

Proof. Taking $P\left(R_{x} \mid i\right)$ from Equation (22), we get

$$
\begin{aligned}
P\left(I_{x}<w \mid R_{x}\right) & =1-\frac{\sum_{i=w}^{\infty} P(i) P\left(R_{x} \mid i\right)}{\sum_{i=0}^{\infty} P(i) P\left(R_{x} \mid i\right)} \\
& =1-\frac{F\left(r_{x}, 0, b\right) \cdot p_{x} \sum_{i=w}^{\infty}\left(1-p_{x}\right)^{i}}{p_{x} \sum_{i=0}^{b-1}\left(1-p_{x}\right)^{i}+F\left(r_{x}, 0, b\right) \cdot p_{x} \sum_{i=b}^{\infty}\left(1-p_{x}\right)^{i}} \\
& =1-\frac{F\left(r_{x}, 0, b\right)\left(1-p_{x}\right)^{w}}{\left(1-\left(1-p_{x}\right)^{b}\right)+F\left(r_{x}, 0, b\right)\left(1-p_{x}\right)^{b}} \\
& =1-\frac{\left(1-p_{x}\right)^{w}}{\frac{1-\left(1-p_{x}\right)^{b}}{F\left(r_{x}, 0, b\right)}+\left(1-p_{x}\right)^{b}} .
\end{aligned}
$$

\subsection{Case $0<\lambda_{x} \leq\left\lceil\frac{w-b}{B}\right\rceil$}

Definition 12. Let $G\left(r_{x}, c_{x}, \lambda_{x}\right)$ be the probability that a specific subset of $c_{x}$ out of $r_{x}$ timers are touched by $B$ inserts, and that the same $c_{x}$ timers are not touched by any of the subsequent $\left(\lambda_{x}-1\right) B+b$ inserts, while the remaining $r_{x}-c_{x}$ timers are touched by those subsequent inserts.

LEMMA 4.2. If $0<\lambda_{x} \leq\left\lceil\frac{w-b}{B}\right\rceil$, then

$$
P\left(R_{x} \mid i\right) \approx \begin{cases}0 & \text { if } i<\left(\lambda_{x}-1\right) B+b \\ G\left(r_{x}, c_{x}, \lambda_{x}\right) & \text { if } i \geq \lambda_{x} B+b \\ F\left(r_{x}, c_{x},\left(\lambda_{x}-1\right) B+b\right) & \text { otherwise. }\end{cases}
$$

Proof. We know that exactly $\left(\lambda_{x}-1\right) B+b$ insertions occurred since $T_{+}$changed from timestamp $T_{x}$ with age $\lambda_{x}$.

CASE $i<\left(\lambda_{x}-1\right) B+b$ : In this case, $x$ would have been inserted since $T_{+}$changed from $T_{x}$, so all timers in $R_{x}$ would have been assigned a timestamp more recent than $T_{x}$. If so, then $\lambda_{x}$ would be less than its observed value, which is a contradiction. Thus, $P\left(R_{x} \mid i\right)=0$.

CASE $i \geq \lambda_{x} B+b$ : In this case, $x$ would have been most recently inserted before $T_{+}=T_{x}$. Thus, the observed $c_{x}, r_{x}$, and $\lambda_{x}$ values must have resulted as follows:

(1) All $c_{x}$ timers in $C_{x}$ were touched by one of the $B$ insertions during which $T_{+}=T_{x}$.

(2) The same $c_{x}$ timers were not touched during the $\left(\lambda_{x}-1\right) B+b$ insertions since $T_{+}=T_{x}$, but the remaining $r_{x}-c_{x}$ timers were touched during those insertions. 
The joint probability of these events is exactly $G\left(r_{x}, c_{x}, \lambda_{x}\right)$, so we have $P\left(R_{x} \mid i\right)=G\left(r_{x}, c_{x}, \lambda_{x}\right)$.

CASE $\left(\lambda_{x}-1\right) B+b \leq i<\lambda_{x} B+b$ : In this case, $x$ would have been most recently inserted while $T_{+}=T_{x}$, so all timers in $R_{x}$ must have been set to $T_{x}$. Thus, $P\left(R_{x} \mid i\right)$ is just the probability $F\left(r_{x}, c_{x},\left(\lambda_{x}-1\right) B+b\right)$ that the $c_{x}$ timers that we observe as still having timestamp $T_{x}$ would not have been overwritten during the last $\left(\lambda_{x}-1\right) B+b$ insertions, and that the remaining $r_{x}-c_{x}$ timers that differ from $T_{x}$ would have been overwritten.

We obtain $G\left(r_{x}, c_{x}, \lambda_{x}\right)$ by finding the probability of each of its constituent events. First, the probability that a particular set of $c_{x}$ timers were touched by one of $B$ insertions is given by $F\left(c_{x}, 0, B\right)$. Second, the probability that the same $c_{x}$ timers were not touched by any of $\left(\lambda_{x}-1\right)$ $B+b$ insertions, while the remaining $r_{x}-c_{x}$ timers were, is given by $F\left(r_{x}, c_{x},\left(\lambda_{x}-1\right) B+b\right)$ ). These two events are largely independent for common BTBF parameters, so we can approximate $G\left(r_{x}, c_{x}, \lambda_{x}\right)$ by multiplying their probabilities,

$$
G\left(r_{x}, c_{x}, \lambda_{x}\right) \approx F\left(c_{x}, 0, B\right) \cdot F\left(r_{x}, c_{x},\left(\lambda_{x}-1\right) B+b\right) .
$$

Computing $P\left(I_{x}<w \mid R_{x}\right)$ is different for $\lambda_{x}=\left\lceil\frac{w-b}{B}\right\rceil$ and $0<\lambda_{x}<\left\lceil\frac{w-b}{B}\right\rceil$, so we handle each separately. In both cases, $P\left(R_{x} \mid i\right)$ is defined as in Equation (24). To shrink equations, we substitute $F(\cdot)$ for $F\left(r_{x}, c_{x},\left(\lambda_{x}-1\right) B+b\right)$ and $G(\cdot)$ for $G\left(r_{x}, c_{x}, \lambda_{x}\right)$. Since $F(\cdot)$ is a term in our approximation for $G(\cdot), G(\cdot) / F(\cdot)$ simplifies to $F\left(c_{x}, 0, B\right)$.

\subsubsection{Case $0<\lambda_{x}<\left\lceil\frac{w-b}{B}\right\rceil$.}

THEorem 4. If $0<\lambda_{x}<\left\lceil\frac{w-b}{B}\right\rceil$, then

$$
P\left(I_{x}<w \mid R_{x}\right)=1-\frac{\left(1-p_{x}\right)^{w-\left(\lambda_{x}-1\right) B-b}}{\frac{1-\left(1-p_{x}\right)^{B}}{F\left(c_{x}, 0, B\right)}+\left(1-p_{x}\right)^{B}} .
$$

Proof. If $\lambda_{x}<\left\lceil\frac{w-b}{B}\right\rceil$, then $\lambda_{x} \leq \frac{w-b}{B}$, and $\lambda_{x} B+b \leq w$. Thus, we can construct the posterior sum as follows:

$$
\begin{aligned}
P\left(I_{x}<w \mid R_{x}\right) & =1-\frac{\sum_{i=w}^{\infty} P(i) P\left(R_{x} \mid i\right)}{\sum_{i=0}^{\infty} P(i) P\left(R_{x} \mid i\right)} \\
& =1-\frac{G(\cdot) \sum_{i=w}^{\infty}\left(1-p_{x}\right)^{i}}{F(\cdot) \sum_{i=\left(\lambda_{x}-1\right) B+b}^{\lambda_{x} B+b-1}\left(1-p_{x}\right)^{i}+G(\cdot) \sum_{i=\lambda_{x} B+b}^{\infty}\left(1-p_{x}\right)^{i}} \\
& =1-\frac{\left(1-p_{x}\right)^{w}}{\frac{F(\cdot)}{G(\cdot)}\left(1-p_{x}\right)^{\left(\lambda_{x}-1\right) B+b}\left(1-\left(1-p_{x}\right)^{B}\right)+\left(1-p_{x}\right)^{\lambda_{x} B+b}} \\
& =1-\frac{\left(1-p_{x}\right)^{w-\left(\lambda_{x}-1\right) B-b}}{\frac{F(\cdot)}{G(\cdot)}\left(1-\left(1-p_{x}\right)^{B}\right)+\left(1-p_{x}\right)^{B}} \\
& =1-\frac{\left(1-p_{x}\right)^{w-\left(\lambda_{x}-1\right) B-b}}{\frac{1-\left(1-p_{x}\right)^{B}}{F\left(c_{x}, 0, B\right)}+\left(1-p_{x}\right)^{B}}
\end{aligned}
$$


4.3.2 Case $\lambda_{x}=\left\lceil\frac{w-b}{B}\right\rceil$.

THeOREM 5. If $\lambda_{x}=\left\lceil\frac{w-b}{B}\right\rceil$, then

$$
P\left(I_{x}<w \mid R_{x}\right)=\frac{1-\left(1-p_{x}\right)^{w-\left(\lambda_{x}-1\right) B-b}}{1-\left(1-p_{x}\right)^{B}\left(1-F\left(c_{x}, 0, B\right)\right)} .
$$

Proof. If $\lambda_{x}=\left\lceil\frac{w-b}{B}\right\rceil$, then $\left(\lambda_{x}-1\right) B+b<w \leq \lambda_{x} B+b$. Thus, we can construct the posterior sum as follows:

$$
\begin{aligned}
P\left(I_{x}<w \mid R_{x}\right) & =\frac{\sum_{i=0}^{w-1} P(i) P\left(R_{x} \mid i\right)}{\sum_{i=0}^{\infty} P(i) P\left(R_{x} \mid i\right)} \\
& =\frac{F(\cdot) \sum_{i=\left(\lambda_{x}-1\right) B+b}^{w-1}\left(1-p_{x}\right)^{i}}{F(\cdot) \sum_{i=\left(\lambda_{x}-1\right) B+b}^{\lambda_{x} B+b-1}\left(1-p_{x}\right)^{i}+G(\cdot) \sum_{i=\lambda_{x} B+b}^{\infty}\left(1-p_{x}\right)^{i}} \\
& =\frac{\left(1-p_{x}\right)^{\left(\lambda_{x}-1\right) B+b}\left(1-\left(1-p_{x}\right)^{w-\left(\lambda_{x}-1\right) B-b}\right)}{\left(1-p_{x}\right)^{\left(\lambda_{x}-1\right) B+b}\left(1-\left(1-p_{x}\right)^{B}\right)+\frac{G(\cdot)}{F(\cdot)}\left(1-p_{x}\right)^{\lambda_{x} B+b}} \\
& =\frac{1-\left(1-p_{x}\right)^{w-\left(\lambda_{x}-1\right) B-b}}{1-\left(1-p_{x}\right)^{B}\left(1-F\left(c_{x}, 0, B\right)\right)} .
\end{aligned}
$$

\subsection{Computing Probabilities Efficiently}

Consider the inferential BTBF's posteriors given by Equations (23), (26), and (27). Since $m$ and $k$ are fixed, $(1-1 / m)^{k}$ can be precomputed for Equation (21). Equation (23) requires $O\left(\log _{2}(w\right.$. $\left.\left.r_{x} \cdot D(b)\right)\right) \leq O\left(\log _{2}(w \cdot k \cdot B)\right)$ multiplications to compute exponents. The cost of computing $D(b)$ depends on the distribution (Section 2.5). Equation (23) is the most expensive, but it is needed only in the rare case when $\lambda_{x}=0$.

Equation (26) needs $O\left(\log _{2} w\right)$ multiplications, and the cost to compute $F\left(c_{x}, 0, B\right)$. As $c_{x}$ takes $O(k)$ values and $B$ is fixed, all $O(k)$ values of $F\left(c_{x}, 0, B\right)$ can be pre-computed and cached. These cached values are used for Equation (27), which requires only $O\left(\log _{2} B\right)$ more multiplications, as $\lambda(x)=\left\lceil\frac{w-b}{B}\right\rceil$. Using these techniques, we spent less time computing probabilities than managing the filter itself.

\section{GENERALIZED BLOOM FILTER}

The Generalized Bloom Filter (GBF) was used in Reference [16] for static set membership queries. As new items are added to the GBF, its memory of older items decays, so it is also well-suited to queries on continuous data streams. Unlike the BTBF, the standard GBF is not built for a particular window width $w$. Over a stream, however, we can still view GBF as responding to sliding window queries. We use it to show how our analysis can be applied to different filter types.

The GBF consists of $k_{0}+k_{1}$ hash functions and an array of $m$ 1-bit cells. GBF notation is summarized in Table 3. 
Table 3. GBF Notation

\begin{tabular}{|l|l|}
\hline$k_{0}, k_{1}$ & Num. hashes that set cells to 0,1 , respectively \\
\hline$R_{x, 0}, R_{x, 1}$ & Cells set by hashes to 0,1, respectively \\
\hline$r_{x, 0}, r_{x, 1}$ & $r_{x, 0}=\left|R_{x, 0}\right|, r_{x, 1}=\left|R_{x, 1}\right|$ \\
\hline$C_{x, 0}, C_{x, 1}$ & Cells in $R_{x, 0}$ set 0 and in $R_{x, 1}$ set 1 , respectively \\
\hline$c_{x, 0}, c_{x, 1}$ & $c_{x, 0}=\left|C_{x, 0}\right|, c_{x, 1}=\left|C_{x, 1}\right|$ \\
\hline$q_{0}, q_{1}, q_{\epsilon}$ & Prob. cell set to 0,1, or not touched, respectively \\
\hline$f_{0}\left(\phi_{0}, j\right)$ & Prob. cell left 0 after $j$ inserts if initially zero with prob. $\phi_{0}$ \\
\hline$C_{d, e}^{s}$ & Coefficient in efficient form of $P\left(I_{x}<w \mid R_{x}\right)$ \\
\hline
\end{tabular}

\subsection{Insert}

To insert item $x$ into the GBF, we do the following:

(1) Set each cell mapped to by the $k_{1}$ hashes to 1 ,

(2) Set each cell mapped to by the $k_{0}$ hashes to 0 .

If a $k_{0}$-hash and a $k_{1}$-hash collide, then the cell is set to 0 . Hence, $R_{x}=R_{x, 0} \cup R_{x, 1}$, with cells $R_{x, 0}=\left\{h_{1}(x), \ldots, h_{k_{0}}(x)\right\}$ set to 0 , and cells $R_{x, 1}=\left\{h_{k_{0}+1}(x), \ldots, h_{k_{0}+k_{1}}(x)\right\} \backslash R_{x, 0}$ set to 1 . Future insertions may set cells in $R_{x, 0}$ to 1 , and cells in $R_{x, 1}$ to 0 , so the filter loses its memory of $x$.

\subsection{Query}

To query a standard GBF for item $x$, we do the following:

(1) Identify $R_{x, 0}$ and $R_{x, 1}$,

(2) Return Pos if and only if every cell in $R_{x, 0}$ is set to 0 and every cell in $R_{x, 1}$ is set to 1 .

False positives and false negatives are both possible. Say we use the GBF for sliding window queries with window width $w$. A false negative occurs when $I_{x}<w$, but a cell in $R_{x, 0}$ is 1 , or a cell in $R_{x, 1}$ is 0 . Such false negatives occur if an item $y$ inserted after $x$ changes one of the cells in $R_{x}$, and that cell is not changed back by a subsequent insert.

A false positive occurs when $I_{x} \geq w$, but all cells in $R_{x, 0}$ are 0 and all in $R_{x, 1}$ are 1 . This happens if (1) later inserts happen to set all cells in $R_{x}$ appropriately, (2) none of the $w$ or more inserts since $x$ change any of the cells in $R_{x}$, leaving them unchanged since $x$ 's last insertion. Combinations of these cases may also generate false positives. For example, a subsequent insert may change a single cell in $R_{x}$, which is later changed back by yet another insert.

Increasing $k_{0}$ or $k_{1}$ increases false negatives, as cells are changed sooner, but reduces false positives, as more cells must be correctly set to respond Pos. Figure 7 demonstrates the standard GBF's operation.

\subsection{GBF Analysis}

Instead of just a binary Pos or NeG, the inferential Generalized Bloom Filter (GBF) returns the sliding window posterior $\lim _{n \rightarrow \infty} P\left(I_{x}<w \mid R_{x}\right)$ in response to queries. We now derive $P\left(I_{x}=j \mid R_{x}\right)$ for the GBF using techniques from Section 2. From this posterior, we derive an expression for the sliding window posterior using Equation (9).

Definition 13. Let $C_{x, 0}$ be the subset of cells in $R_{x, 0}$ that are set to 0 , and $C_{x, 1}$ the subset of $R_{x, 1}$ set to 1 .

Let $r_{x, 0}=\left|R_{x, 0}\right|, r_{x, 1}=\left|R_{x, 1}\right|, c_{x, 0}=\left|C_{x, 0}\right|, c_{x, 1}=\left|C_{x, 1}\right|$. The values $c_{x, 0}$ and $c_{x, 1}$ indicate how many of the $r_{x, 0}$ and $r_{x, 1}$, respectively, are set as they would be if $x$ had just been inserted. 


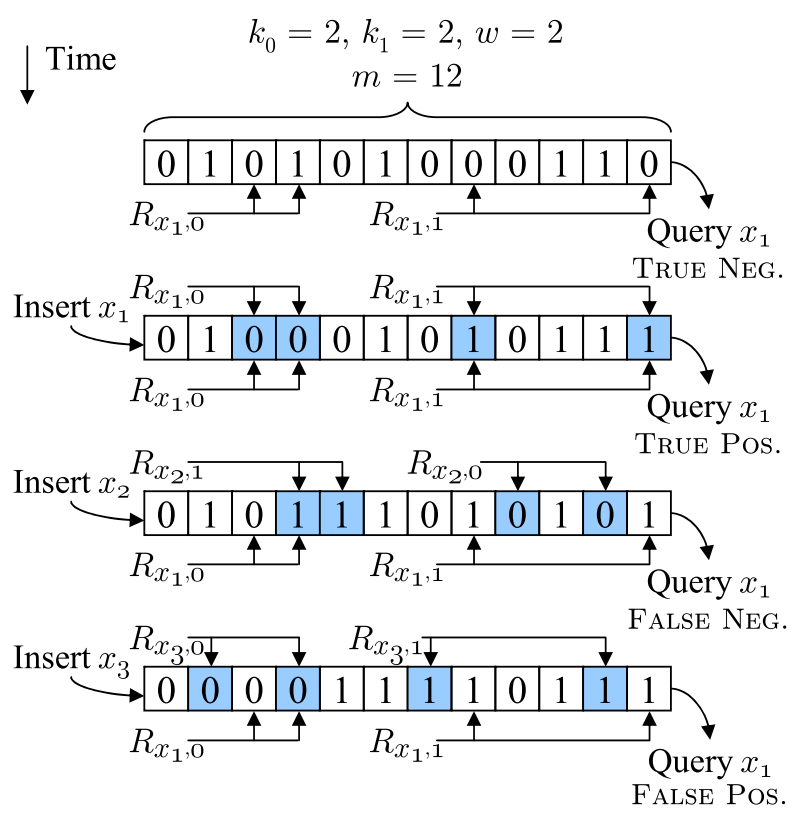

Fig. 7. Operation of a standard GBF. Cells touched by insertions shaded.

Intuitively, the larger $c_{x, 0}$ and $c_{x, 1}$, the higher the probability that $x$ was recently inserted. Notation for the GBF analysis is summarized in Table 3.

5.3.1 Computing Probabilities. We want $\lim _{n \rightarrow \infty} P\left(I_{x}<w \mid R_{x}\right)$, given $\lim _{n \rightarrow \infty} P(j)$ from Equation (4). Again, we omit the limit notation for brevity. We first find an expression for $P\left(R_{x} \mid j\right)$, which can be used in Equation (9) or (10) to find $P\left(I_{x}<w \mid R_{x}\right)$.

Let $q_{0}$ be the probability that at least one of the $k_{0}$ hashes sets a given cell to 0 :

$$
q_{0}=1-\left(1-\frac{1}{m}\right)^{k_{0}}
$$

Similarly, let $q_{1}$ be the probability that at least one of the $k_{1}$ hashes, but none of the $k_{0}$ hashes, mapped to a given cell:

$$
q_{1}=\left(1-\left(1-\frac{1}{m}\right)^{k_{1}}\right)\left(1-\frac{1}{m}\right)^{k_{0}} .
$$

Let $q_{\epsilon}$ be the probability that no hash maps to a given cell:

$$
q_{\epsilon}=1-q_{0}-q_{1}=\left(1-\frac{1}{m}\right)^{k_{0}+k_{1}} .
$$

THEOREM 6. Let $f_{0}\left(\phi_{0}, i\right)$ denote the probability that a cell contains a 0 after $i$ insertions, given that it was initially 0 with probability $\phi_{0} . f_{0}\left(\phi_{0}, i\right)$ is given by

$$
f_{0}\left(\phi_{0}, i\right)=\phi_{0} q_{\epsilon}^{i}+\frac{q_{0}}{q_{0}+q_{1}}\left(1-q_{\epsilon}^{i}\right)
$$

Proof. A cell can contain a 0 after $i$ insertions in two cases. First, if it contained a 0 before the insertions and was not touched during any of the $i$ inserts, with probability $\phi_{0} q_{\epsilon}^{i}$. Second, if the cell was set to 0 by an insert, then not touched by any subsequent inserts, with probability $\sum_{\ell=0}^{i-1} q_{0} q_{\epsilon}^{\ell}$. 
Since these events are independent,

$$
\begin{aligned}
f_{0}\left(\phi_{0}, i\right) & =\phi_{0} q_{\epsilon}^{i}+\sum_{\ell=0}^{i-1} q_{0} q_{\epsilon}^{\ell}=\phi_{0} q_{\epsilon}^{i}+q_{0} \frac{1-q_{\epsilon}^{i}}{1-q_{\epsilon}} \\
& =\phi_{0} q_{\epsilon}^{i}+\frac{q_{0}}{q_{0}+q_{1}}\left(1-q_{\epsilon}^{i}\right) .
\end{aligned}
$$

A parallel analysis appears in Reference [16] for false-positive and false-negative rates. We now use it to approximate $P\left(R_{x} \mid i\right)$. When $x$ was inserted, all $r_{x, 0}$ cells in $R_{x, 0}$ were set to 0 , and all $r_{x, 1}$ cells in $R_{x, 1}$ set to 1 , so we know each cell's starting value. $P\left(R_{x} \mid i\right)$ is the probability that all cells in $R_{x, 0}$ and $R_{x, 1}$ are set as we observe them, given that $i$ items have been inserted since $x$ was last inserted. Our observations imply that the following events occurred over the $i$ insertions:

\begin{tabular}{l|l}
\hline Events & Prob. Each \\
\hline$c_{x, 0}$ cells that started 0 remained 0 & $f_{0}(1, i)$ \\
\hline$r_{x, 0}-c_{x, 0}$ cells that started as 0 changed to 1 & $\left(1-f_{0}(1, i)\right)$ \\
\hline$c_{x, 1}$ cells that started as 1 remained 1 & $\left(1-f_{0}(0, i)\right)$ \\
\hline$r_{x, 1}-c_{x, 1}$ cells that started as 1 changed to 0 & $f_{0}(0, i)$ \\
\hline
\end{tabular}

We can approximate $P\left(R_{x} \mid i\right)$ by treating these events as independent and taking the product of their probabilities:

$$
\begin{aligned}
P\left(R_{x} \mid i\right) \approx f_{0}(1, i)^{c_{x, 0}} \times\left(1-f_{0}(1, i)\right)^{r_{x, 0}-c_{x, 0}} \\
\\
\times\left(1-f_{0}(0, i)\right)^{c_{x, 1}} \times f_{0}(0, i)^{r_{x, 1}-c_{x, 1}}
\end{aligned}
$$

We can use $P\left(R_{x} \mid i\right)$ from Equation (32) and $P(i)$ from Equation (4) in Equation (9) to get $P\left(I_{x}<\right.$ $\left.w \mid R_{x}\right)$. The resulting infinite sum having no closed form, we use approximations.

5.3.2 Computing Probabilities Efficiently. To utilize space efficiently, the numbers of 0-cells and 1 -cells must be balanced, so $k_{0}=k_{1}=k$. Now,

$$
\begin{aligned}
\frac{q_{0}}{q_{0}+q_{1}} & =\frac{1}{1+\frac{q_{1}}{q_{0}}} \approx \frac{1}{1+\frac{\left(1-\left(1-\frac{1}{m}\right)^{k}\right)\left(1-\frac{1}{m}\right)^{k}}{\left(1-\left(1-\frac{1}{m}\right)^{k}\right)}} \\
& =\frac{1}{1+\left(1-\frac{1}{m}\right)^{k}} .
\end{aligned}
$$

When $m$ is large, as is common, we get $\left(1-\frac{1}{m}\right)^{k} \approx 1$, and $\frac{q_{0}}{q_{0}+q_{1}} \approx \frac{1}{2}$. Thus, Equation (31) reduces to

$$
f_{0}\left(\phi_{0}, i\right) \approx \phi_{0} q_{\epsilon}^{i}+\frac{1}{2}\left(1-q_{\epsilon}^{i}\right)=q_{\epsilon}^{i}\left(\phi_{0}-\frac{1}{2}\right)+\frac{1}{2} .
$$

Thus, Equation (32) reduces as follows:

$$
\begin{aligned}
P\left(R_{x} \mid i\right) \approx & f_{0}(1, i)^{c_{x, 0}} \times\left(1-f_{0}(1, i)\right)^{r_{x, 0}-c_{x, 0}} \\
& \times\left(1-f_{0}(0, i)\right)^{c_{x, 1}} \times f_{0}(0, i)^{r_{x, 1}-c_{x, 1}} \\
= & \left(\frac{1+q_{\epsilon}^{i}}{2}\right)^{c_{x, 0}} \times\left(\frac{1-q_{\epsilon}^{i}}{2}\right)^{r_{x, 0}-c_{x, 0}}
\end{aligned}
$$




$$
\begin{gathered}
\times\left(\frac{1+q_{\epsilon}^{i}}{2}\right)^{c_{x, 1}} \times\left(\frac{1-q_{\epsilon}^{i}}{2}\right)^{r_{x, 1}-c_{x, 1}} \\
=\left(\frac{1-q_{\epsilon}^{i}}{2}\right)^{r_{x, 0}+r_{x, 1}} \times\left(\frac{1+q_{\epsilon}^{i}}{1-q_{\epsilon}^{i}}\right)^{c_{x, 0}+c_{x, 1}} .
\end{gathered}
$$

Now we apply Equation (9) to get

$$
\begin{aligned}
P\left(I_{x}<w \mid R_{x}\right) & =\frac{\sum_{i=0}^{w-1} P(i) P\left(R_{x} \mid i\right)}{\sum_{i=0}^{\infty} P(i) P\left(R_{x} \mid i\right)} \\
& =\frac{\sum_{i=0}^{w-1} p_{x}\left(1-p_{x}\right)^{i}\left(\frac{1-q_{\epsilon}^{i}}{2}\right)^{r_{x, 0}+r_{x, 1}} \times\left(\frac{1+q_{\epsilon}^{i}}{1-q_{\epsilon}^{i}}\right)^{c_{x, 0}+c_{x, 1}}}{\sum_{i=0}^{\infty} p_{x}\left(1-p_{x}\right)^{i}\left(\frac{1-q_{\epsilon}^{i}}{2}\right)^{r_{x, 0}+r_{x, 1}} \times\left(\frac{1+q_{\epsilon}^{i}}{1-q_{\epsilon}^{i}}\right)^{c_{x, 0}+c_{x, 1}}} \\
& =\frac{\sum_{i=0}^{w-1}\left(1-p_{x}\right)^{i}\left(1-q_{\epsilon}^{i}\right)^{r_{x, 0}+r_{x, 1}} \times\left(\frac{1+q_{\epsilon}^{i}}{1-q_{\epsilon}^{i}}\right)^{c_{x, 0}+c_{x, 1}}}{\sum_{i=0}^{\infty}\left(1-p_{x}\right)^{i}\left(1-q_{\epsilon}^{i}\right)^{r_{x, 0}+r_{x, 1}} \times\left(\frac{1+q_{\epsilon}^{i}}{1-q_{\epsilon}^{i}}\right)^{c_{x, 0}+c_{x, 1}}} .
\end{aligned}
$$

We turn to Equation (33). Let us substitute $d=c_{x, 0}+c_{x, 1}$ and $e=r_{x, 0}+r_{x, 1}-d$. In general, we want the sum:

$$
\Psi_{\eta}=\sum_{i=0}^{\eta-1}\left(1-p_{x}\right)^{i}\left(1+q_{\epsilon}^{i}\right)^{d}\left(1-q_{\epsilon}^{i}\right)^{e} .
$$

Using the binomial theorem, we can re-write:

$$
\left(1+q_{\epsilon}^{i}\right)^{d}\left(1-q_{\epsilon}^{i}\right)^{e}=\sum_{s=0}^{d+e} C_{d, e}^{s}\left(q_{\epsilon}^{i}\right)^{s}
$$

where $C_{d, e}^{s}$ is the coefficient of $\left(q_{\epsilon}^{i}\right)^{s}$ from the convolution

$$
C_{d, e}^{s}=\sum_{\ell=0}^{s}\left(\begin{array}{l}
d \\
\ell
\end{array}\right)(-1)^{s-\ell}\left(\begin{array}{c}
e \\
s-\ell
\end{array}\right)=\sum_{\ell=0}^{s}\left(\begin{array}{c}
d \\
s-\ell
\end{array}\right)(-1)^{\ell}\left(\begin{array}{l}
e \\
\ell
\end{array}\right),
$$

where $\left(\begin{array}{l}a \\ b\end{array}\right)=0$ if $b>a$. Now we can re-write $\Psi_{\eta}$ as

$$
\begin{aligned}
\Psi_{\eta} & =\sum_{i=0}^{\eta-1}\left(1-p_{x}\right)^{i}\left(\sum_{s=0}^{d+e} C_{d, e}^{s}\left(q_{\epsilon}^{i}\right)^{s}\right) \\
& =\sum_{s=0}^{d+e} C_{d, e}^{s} \sum_{i=0}^{\eta-1}\left(\left(1-p_{x}\right) q_{\epsilon}^{s}\right)^{i} \\
& =\sum_{s=0}^{d+e} C_{d, e}^{s} \frac{1-\left(\left(1-p_{x}\right) q_{\epsilon}^{s}\right)^{\eta}}{1-\left(1-p_{x}\right) q_{\epsilon}^{s}} .
\end{aligned}
$$

Applying to Equation (33), we get

$$
\begin{aligned}
P\left(I_{x}<w \mid R_{x}\right) & =\frac{\Psi_{w}}{\lim _{\eta \rightarrow \infty} \Psi_{\eta}} \\
& =\frac{\sum_{s=0}^{d+e} C_{d, e}^{s} \frac{1-\left(\left(1-p_{x}\right) q_{\epsilon}^{s}\right)^{w}}{1-\left(1-p_{x}\right) q_{\epsilon}^{s}}}{\sum_{s=0}^{d+e} C_{d, e}^{s} \frac{1}{1-\left(1-p_{x}\right) q_{\epsilon}^{s}}} .
\end{aligned}
$$


This is easy to evaluate. First, $d+e=r_{x, 0}+r_{x, 1} \leq 2 k$, so we need to sum at most $2 k$ terms, with $k$ being usually small. We also have $d, e, s \leq 2 k$, so there are at most $8 k^{3}$ distinct coefficients $C_{d, e}^{s}$, which we can easily pre-compute. It is also unlikely that $r_{x, 0}+r_{x, 1}$ is much less than $2 k$, so only $O(k)$ combinations of $d, e$ occur in practice, reducing the number of precomputed coefficients to $O\left(k^{2}\right)$. In our experiments we precompute at most $16 k^{2}$ coefficients, which is manageable.

Equation (34) is accurate only for relatively small values of $k$. As $k$ grows, the coefficients $C_{d, e}^{s}$ and the corresponding terms can have large absolute values, though their sum must lie between 0 and 1. If precision is limited and $k$ is large, then roundoff error can occur. Using double-precision floating point numbers, such roundoff error leads to posterior probabilities outside the $[0,1]$ range for approximately $k \geq 30$.

An advantage of the inferential GBF over the inferential BTBF is that we can vary $w$ at any time, whereas $w$ for the BTBF must be fixed up-front. We could improve the accuracy of the inferential GBF by replacing $i$ in Equation (31) with $D(i)$ (Equation (15)), as we did for the inferential BTBF. However, doing so would prevent us from constructing the efficiently computable expression in Equation (34).

\section{EXPERIMENTS}

\subsection{Experimental Setup}

We examined four approaches for sliding window queries: the standard GBF and BTBF, which return Pos or NEG, and the inferential GBF and BTBF, which return the sliding window posterior $P\left(I_{x}<w \mid R_{x}\right)$. We now test if using posteriors reduces overall penalties, when penalties for false positives and negatives vary across queries. Our experiments use a real-world data stream and two synthetic data streams.

6.1.1 Queries and Error Penalties. We use the same data stream for queries and inserts. As each new item $x$ arrives, we always query for $x$ and then insert $x$. This model might be used for an expensive multi-level LRU cache, where we only want to do an expensive check of a large cache level if we are likely to find the item. This model also resembles duplicate detection as used for mitigating click fraud $[15,38]$, although duplicates would not be inserted in that case.

Let $\$_{F P}$ and $\$_{F N}$ be the penalties incurred if the filter makes false-positive/false-negative errors, respectively. We choose $\$_{F P}$ and $\$_{F N}$ independently and uniformly at random from the range $[1.0,10.0)$ for each query. The inferential BTBF uses the minimum expected penalty strategy described in Section 2.6 for deciding whether to return Pos or NEG.

6.1.2 Parameter Selection. Poor choices for filter parameters lead to more errors. However, there is no consensus on how to choose parameters for the BTBF, though References [16, 33] provide limited guidance. For the GBF, we fix $k_{0}=k_{1}=k$. For the BTBF, we fix $b p t=k$ as in Reference [33]. If $k<3$, then we set the minimum $b p t=3$ needed by the BTBF. Given $k$, we choose the smallest $\mathcal{P}$ that allows us to check at most $k$ timers per insertion.

Our focus is not on predicting optimal parameters, so we tried all $k$ for $1 \leq k \leq 30$ for each trial, chosing $k$ to minimize total penalty (see Figure 8). Thus, penalties measured for each filter are independent of the parameter selection mechanism. This way, we are able to ensure that a suboptimal parameter selection strategy does not adversely impact any filter's reported performance.

In the GBF, bits set by one of the $k_{0}$ and $k_{1}$ hashes are set to 0 , so the GBF would benefit from separately optimizing $k_{0}$ and $k_{1}$, allowing for a $k_{0}$ slightly less than $k_{1}$ [16]. For simplicity, we do not optimize them separately here. The standard GBF is prone to false negatives if $k$ is large, so its optimal $k$ are small. However, optimal $k$ are larger for the inferential GBF, which uses the added information available with large $k$. 


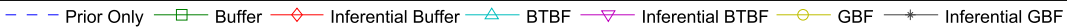

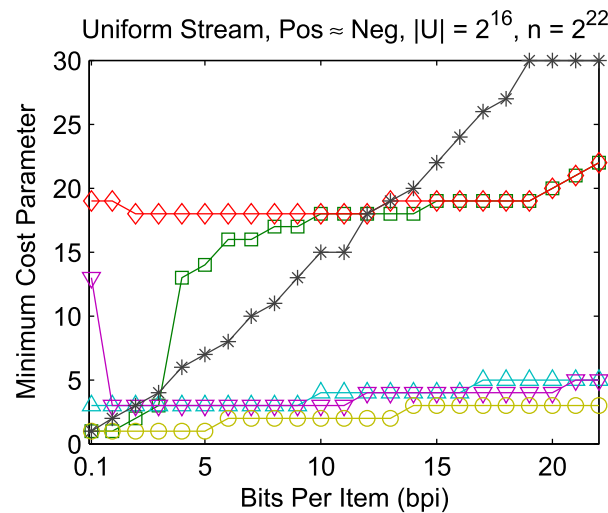

(a) Uniform Stream, $[\mathrm{POS} \approx \mathrm{NEG}]$.

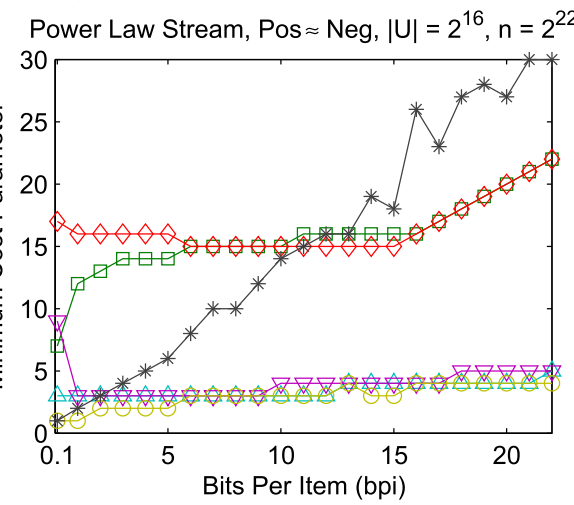

(c) Power Law Stream, $[\mathrm{Pos} \approx \mathrm{Neg}]$.

IP Source Stream, Pos $\approx \mathrm{Neg},|\mathrm{U}|=2^{32}, \mathrm{n}=2^{22}$

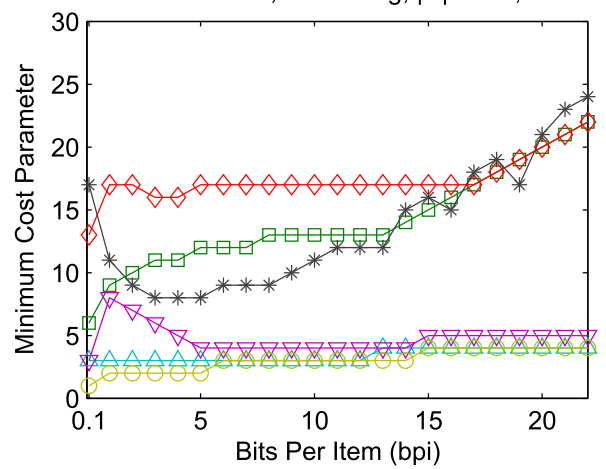

(e) IP Source Stream, $[\mathrm{Pos} \approx \mathrm{NEG}]$.

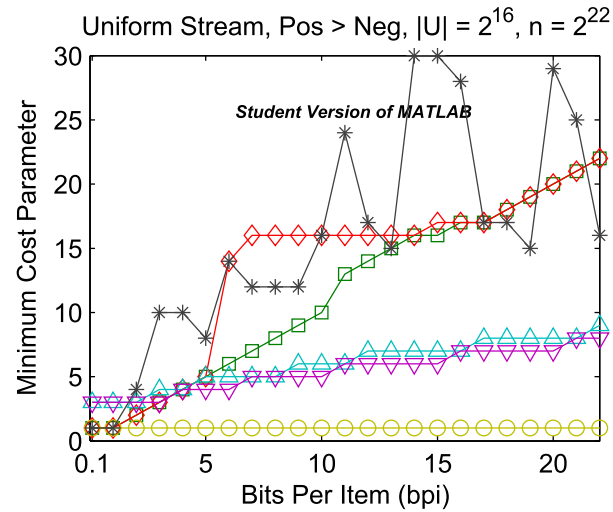

(b) Uniform Stream, [Pos $>$ NeG].

Power Law Stream, Pos $>$ Neg, $|\mathrm{U}|=2^{16}, n=2^{22}$

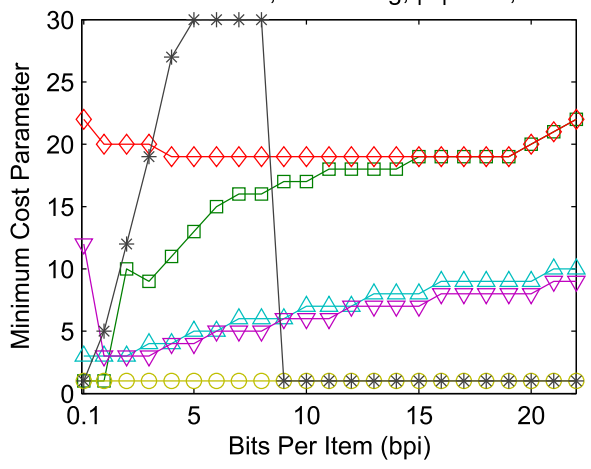

(d) Power Law Stream, [Pos $>$ Neg].

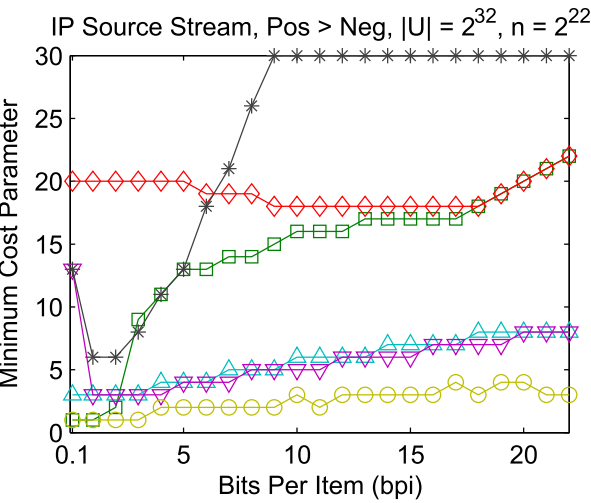

(f) IP Source Stream, [Pos $>$ NeG] .

Fig. 8. Minimum cost parameters ( $k$ for BTBF/GBF, $b p h$ for Simple Buffer) in each experiment suite. 
Table 4. Data Parameters and Characteristics for Each Experiment/Condition

\begin{tabular}{|l|c|c|c|c|}
\hline Stream & $|U|$ & Condition & $w$ & Pos/NEG \\
\hline Uniform & $2^{16}$ & {$[$ Pos $\approx$ NEG $]$} & $2^{16}$ & 1.718 \\
\hline Uniform & $2^{16}$ & {$[$ Pos $>$ NEG $]$} & $2^{18}$ & 53.547 \\
\hline Power Law & $2^{16}$ & {$[$ Pos $\approx$ NEG $]$} & $2^{11}$ & 1.182 \\
\hline Power Law & $2^{16}$ & {$[$ Pos $>$ NEG $]$} & $2^{18}$ & 13.451 \\
\hline IP Source & $2^{32}$ & {$[$ Pos $\approx$ NEG $]$} & $2^{8}$ & 1.171 \\
\hline IP Source & $2^{32}$ & {$[$ Pos $>$ NEG $]$} & $2^{15}$ & 8.893 \\
\hline
\end{tabular}

Pos/Neg gives the ratio of queries for items with $I_{x}<w$ to those with $I_{x} \geq w$.

6.1.3 Measuring Performance for Each Filter. Each filter is given bpi bits per item in the window, so the total space is $w \cdot b p i$ bits. Each experimental trial measures the total penalty incurred by a given filter for a specific data stream, choice of $w$, and choice of $b p i$. Each trial over a given stream uses the same sequence of $n=2^{22}$ item inserts/queries, and the same sequence of penalties $\$_{F P}$ and $\$_{F N}$, ensuring comparable results. Before each trial, all cells in the BTBF are set to $T_{\varepsilon}$. We then insert $2^{20}$ items without issuing queries, initializing the filters with "past" items from the data stream.

An experiment is a group of trials with the same stream and $w$, and measures penalties incurred by the standard and inferential BTBF for a range of $b p i$ values. For each stream, we used two experimental conditions. Condition [Pos $\approx \mathrm{NEG}$ ] uses a small enough $w$ to make the numbers of queries requiring Pos and NEg responses roughly the same. Condition [Pos $>$ NEG] uses a larger $w$, so Pos queries outnumber NEg ones.

Each experiment is shown as a single curve on a graph. Some graphs show the total penalty over the trial, while others show the penalty ratio, which is the ratio of the total penalty of the inferential filter over that of the corresponding standard one. A penalty ratio under $100 \%$ indicates that the inferential filter outperformed the standard one.

The choice of $w$ and the Pos/Neg ratios for each experiment are given in Table 4. For simplicity, we chose $w$ to be a power of 2, but our implementation supports arbitrary integral $w$ values.

6.1.4 Errors from Approximations. We made several approximations while deriving the posterior $P\left(I_{x}<w \mid R_{x}\right)$, so we evaluate its accuracy for each dataset. In each experiment, we group queries into 20 bins based on the posterior value $P$ returned. The first bin contains queries with $0 \leq P<0.05$, the second with $0.05 \leq P<0.1$, on up to the last with $0.95 \leq P \leq 1$. Let $\eta_{\ell}$ be the number of queries in bucket $\ell$, and let $M_{\ell}$ be the midpoint of bucket $\ell$. We let $f_{\ell}$ be the fraction of queries in bucket $\ell$ for which $I_{x}<w$. Without approximations, we should have $f_{\ell} \approx M_{\ell}$ for all $\ell$.

Posterior Error, defined as the average absolute difference between a query posterior and its bucket midpoint, equals

$$
\frac{1}{n} \sum_{\ell=1}^{20}\left|f_{\ell}-M_{\ell}\right| \cdot \eta_{\ell} .
$$

Some Posterior Error is unavoidable due to the coarseness of our grouping. Thus, we expect a baseline error of less than half the bucket width (0.025). We graph Posterior Errors for each experiment below.

6.1.5 Implementation. We implemented the filters in Java, running each trial as a single thread on a $2.4 \mathrm{GHz}$ processor. The average time to query and insert an item fell between 0.5 and $1.5 \mu \mathrm{s}$ for the standard and inferential BTBF. Table 5 shows the average time to do an insert/query pair for each decision technique, with a fixed $k=4, b p i=15, w=2^{16}$ for all trials. Times were measured 
Table 5. Average Time per Insert/Query on the Uniform [Pos $\approx$ NEG] Data Stream with $b p i=10$ and $k=4$

\begin{tabular}{|l|c|}
\hline Technique & Time per Query $(\mu \mathrm{s})$ \\
\hline \hline Prior Only & 0.33 \\
\hline BTBF (Standard) & 0.80 \\
\hline BTBF (Inferential) & 1.13 \\
\hline GBF (Standard) & 1.64 \\
\hline GBF (Inferential) & 1.85 \\
\hline
\end{tabular}

for the Uniform data stream (see Section 6.3) and averaged over all $n$ items; times for other streams and window sizes were comparable.

Increasing bpi primarily increases the number of cells, which should have negligible effect on runtime as long as the filter still fits in RAM. Time varies roughly linearly with $k$; the GBF evaluates $2 k$ hashes for each insert/query, so its times are double those of the BTBF. Hashing is done separately for query and insert. Times can be reduced using optimized or hardware implementations.

The inferential BTBF caches $O(k)$ static floating-point values to speed up computation in common cases (Section 4.4), and the GBF caches $O\left(k^{2}\right)$ static floating-point values (Section 5.3.2). Cache sizes are substantial only for a GBF with small $w$, small $b p i$, and large $k$, so we do not count caches as part of the space consumed by our filters.

\subsection{Simple Buffer}

One might ask under what circumstances space devoted to filters could be better spent on a list of $b p h$-bit hashes of the last $\delta \leq w$ items inserted. The Simple Buffer is a contrived decision technique that performs queries via a linear scan over such a list. Practical techniques based on such a list may index it using a Counting Bloom Filter $[13,35,36]$ or a hash table. If the GBF or BTBF incurs lower costs than (outperforms) the Simple Buffer, then it also outperforms these other techniques, which store the list and the index.

Storing all items in the window $(\delta=w, b p h=b p i)$ is impractical when $b p i$ is small, as all $2^{b p i}$ hash values will be in the buffer with high probability. Thus, for each trial, we tried all $b p h$ for $1 \leq b p h \leq 30$, and chose the value of $b p h$ that minimized total cost, with $\delta=\lfloor w \cdot b p i / b p h\rfloor($ see Figure 8). Once $b p i$ is large enough to uniquely represent each item in $U$ or in the window ( $b p i \approx$ $\left.\log _{2} \operatorname{Min}(w,|U|)\right)$, the Simple Buffer achieves near-perfect accuracy.

We can analyze the Simple Buffer using the framework described in Section 2. Let $R_{x}=1$ if the $b p h$-bit hash of $x$ is in the buffer, and $R_{x}=0$, otherwise. The posterior is given by

$$
P\left(I_{x}<w \mid R_{x}\right)= \begin{cases}1-\left(1-p_{x}\right)^{w-\delta} & R_{x}=0 \\ 1-\frac{\left(1-p_{x}\right)^{w}}{\frac{1-\left(1-p_{x}\right)^{\delta}}{1-\left(1-\left(\frac{1}{2}\right)^{b p h}\right)^{D(\delta)}}+\left(1-p_{x}\right)^{\delta}} & R_{x}=1 .\end{cases}
$$

If a technique outperforms the inferential Simple Buffer, then it also outperforms inferential listbased techniques.

\subsection{Uniform Data Stream}

The Uniform data stream samples uniformly with replacement, from a set $U$ of $2^{16}$ integers. $p_{x}=1 /|U|$ forall $x \in U . D(j)$ is given by Equation (16). Figure 9(a) shows Penalty ratios, and 


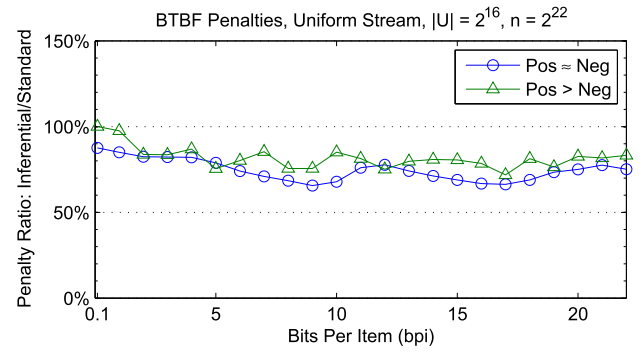

(a) Penalty Ratios, Uniform Stream.

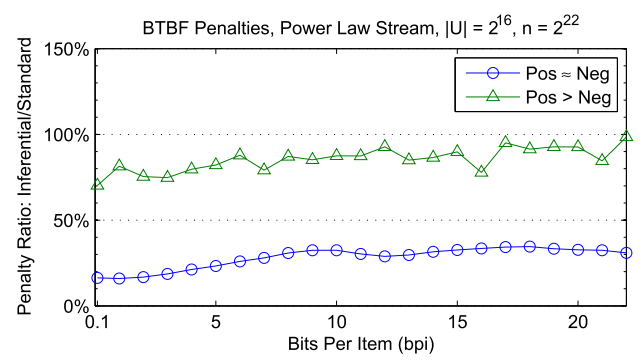

(c) Penalty Ratios, Power Law Stream.

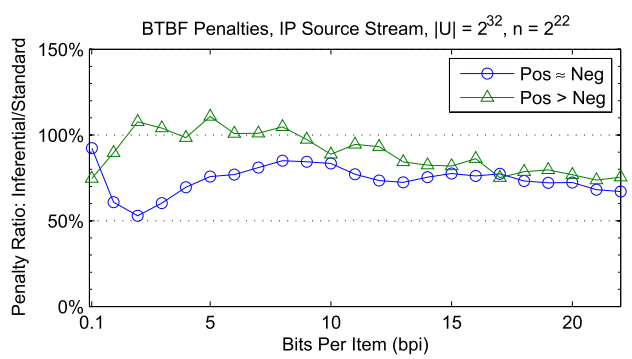

(e) Penalty Ratios, IP Source Stream.

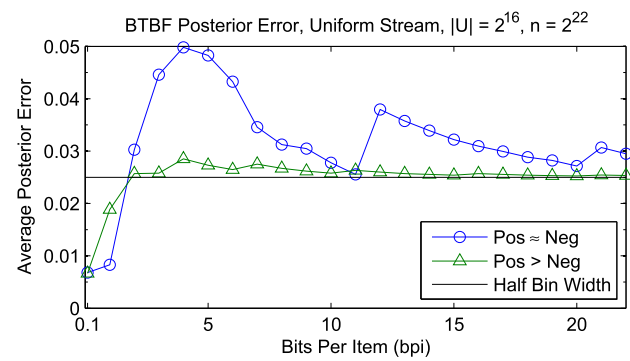

(b) Posterior Errors, Uniform Stream.

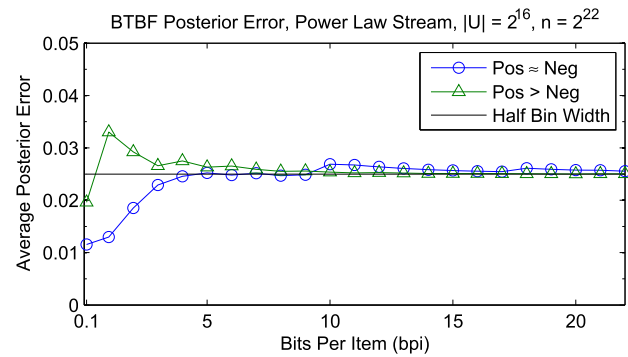

(d) Posterior Errors, Power Law Stream.

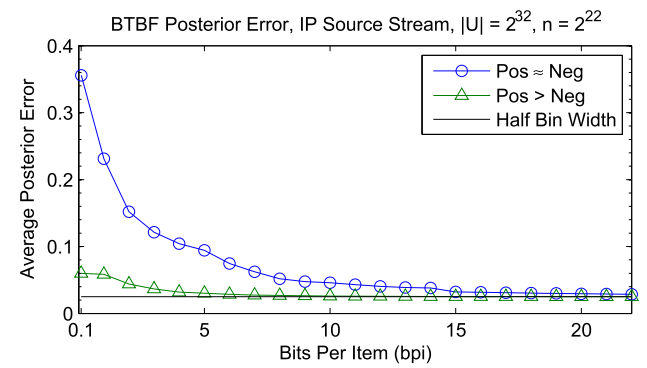

(f) Posterior Errors for IP Source stream.

Fig. 9. BTBF performance for various stream types. Lower $Y$ values are better.

Figure 9(b) shows Posterior Errors. At very low bpi all the filters hold little information, so posteriors depend primarily on the prior $P\left(I_{x}=i\right)$. Since the prior is known exactly, the posterior here is quite accurate.

Penalties for the inferential BTBF are about $80 \%$ of those for standard BTBF. For large bpi, so much state information is available that most posteriors are close to 0 or 1 . They differ from their corresponding bin centers by half the bin width, hence the convergence to 0.025 for the BTBF. Our approximations produce noteworthy error in the BTBF only for moderate $b p i$. The Posterior Errors for such bpi remain under 0.05, indicating largely accurate posterior expressions.

The inferential GBF consistently outperforms the standard GBF (see Figure 10(a)), but both perform poorly overall. For the GBF under [Pos $\approx \mathrm{NEG}$ ], posterior error increases steadily with bpi (see Figure 10(b)).

Figures 11(a) and 11(b) show that in both suites, the inferential BTBF outperforms all other techniques until $b p i \approx 20$, where the Simple Buffer can afford nearly unique representations of all items in the window. One exception occurs in the $[\mathrm{Pos} \approx \mathrm{NEG}]$ suite at $b p i=1$, where the inferential 


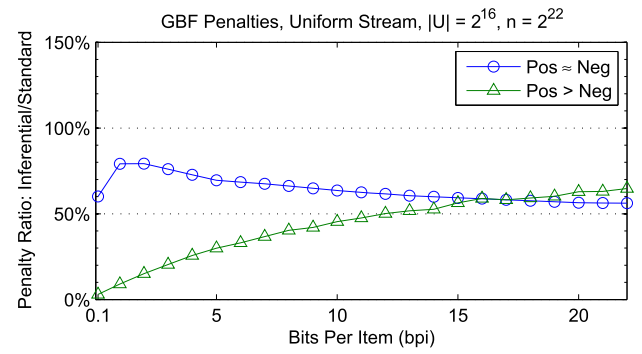

(a) GBF Penalty Ratios, Uniform Stream.

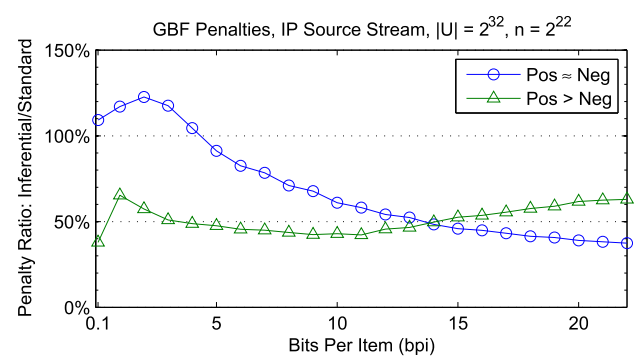

(c) GBF Penalty Ratios, Power Law Stream.

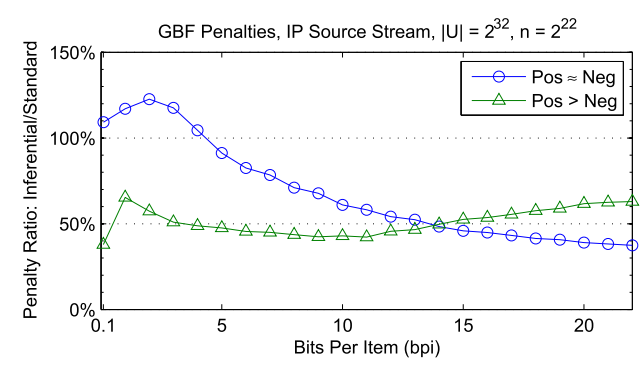

(e) GBF Penalty Ratios, IP Source Stream.

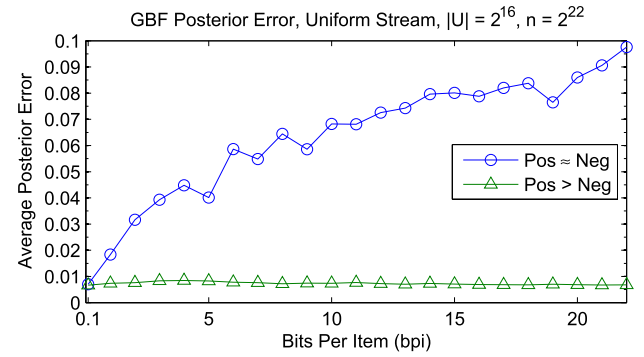

(b) GBF Posterior Errors, Uniform Stream.

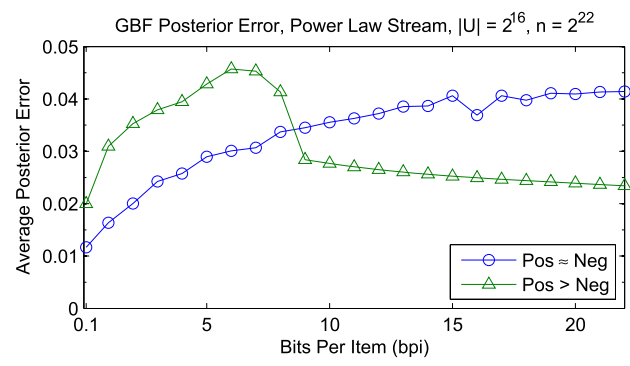

(d) GBF Posterior Errors, Power Law Stream.

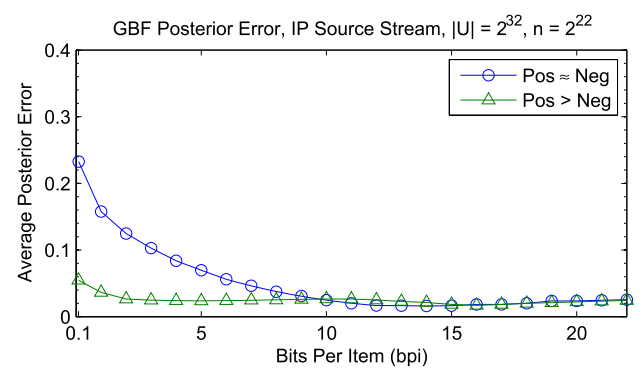

(f) GBF Posterior Errors for IP Source stream.

Fig. 10. GBF performance for various stream types. Lower $Y$ values are better.

GBF beats the BTBF by a small margin. For the [Pos $\approx$ NEG] suite, $k$ reaches our maximum value 30 at $b p i=19$ (see Figure 8), indicating that larger $k$, though inefficient, may yield better results for higher $b p i$.

\subsection{Streams with Skewed Distributions}

For skewed stream item distributions, computing accurate posteriors requires the following:

Assumption 1. $p_{x}$ is easy to compute for each $x$.

Assumption 2. $p_{x}$ is time-invariant for each $x$.

If Assumption 1 is violated, then priors, and thus posteriors, cannot be computed efficiently. If Assumption 2 is violated, then the time-invariant priors yield inaccurate posteriors, increasing penalties.

We must distinguish between $x$ 's value and its rank. More frequent items (larger $p_{x}$ ) have lower rank. Often, item ranks follow a predictable distribution, such as a power law in the case of 


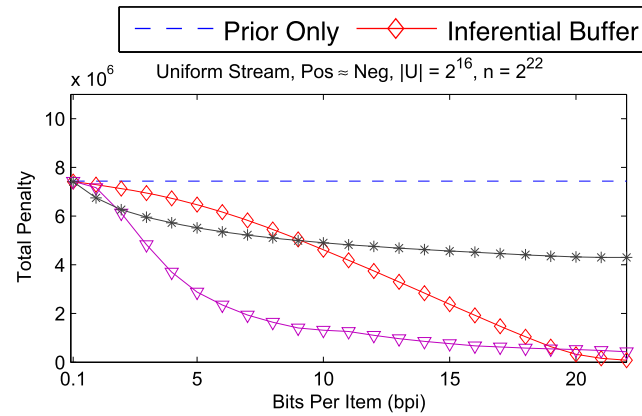

(a) Uniform Stream $[\mathrm{Pos} \approx \mathrm{NeG}]$.

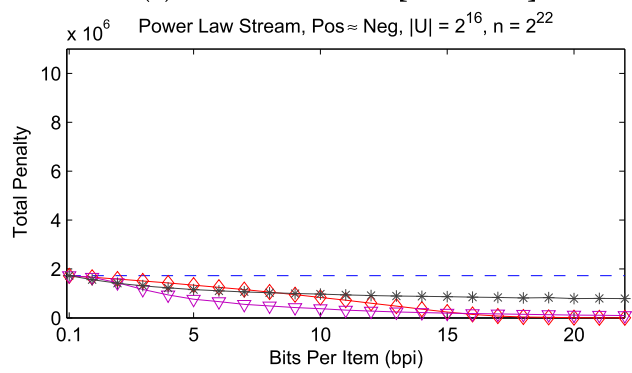

(c) Power Law Stream, $[\mathrm{Pos} \approx \mathrm{NEG}]$.

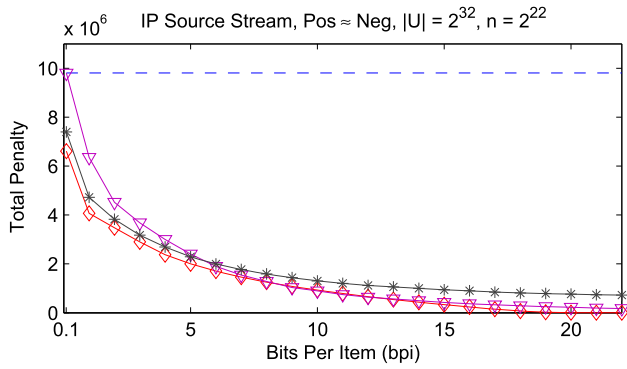

(e) IP Source Stream, $[\mathrm{Pos} \approx \mathrm{NeG}]$.

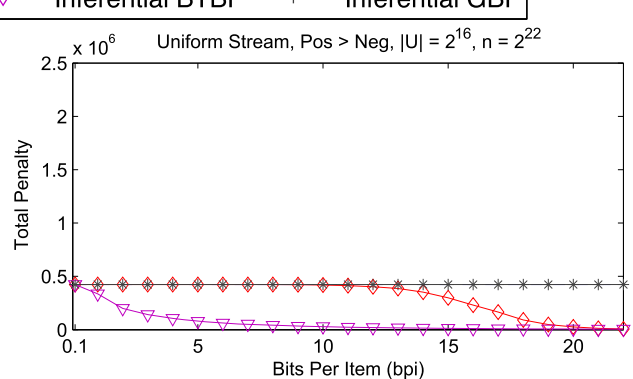

(b) Uniform Stream [Pos $>\mathrm{NeG}]$.

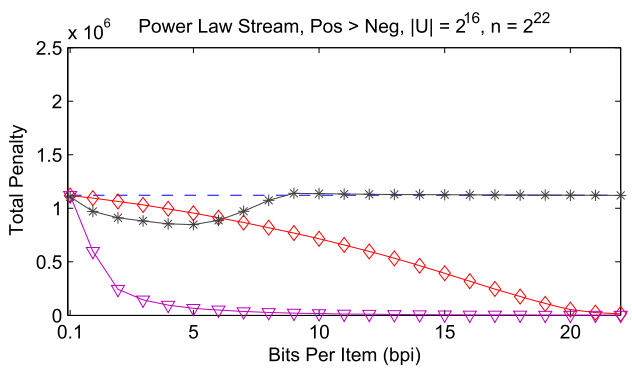

(d) Power Law Stream, [Pos $>$ NeG].

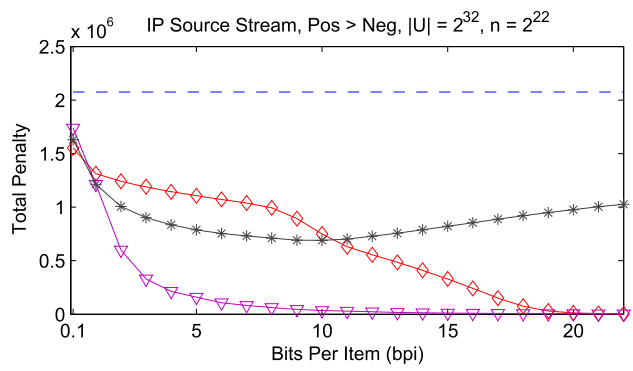

(f) IP Source Stream, [Pos $>$ NEG].

Fig. 11. Total Penalty values for different input stream types and parameters. Lower $Y$ values are better.

Zipf-distributed data, while values do not. Thus, this property only helps compute $p_{x}$ if we can infer $x$ 's rank from its value, which is often not the case.

6.4.1 Power Law Stream. Our Power Law stream samples items from set $U=\{x \in \mathbb{Z} \mid 1 \leq x \leq$ $\left.2^{16}\right\}$ according to the discrete power law distribution $p_{x}=1 /\left(x \cdot H_{|U|}\right)$, where $H_{|U|}=\sum_{i=1}^{|U|} 1 / i$. Computing $p_{x}$ is easy, since $H_{|U|}$ is fixed, and $p_{x}$ is time-invariant. Equation (17) gives $D(j)$.

BTBF penalty ratios are shown in Figure 9(c), and Posterior Errors in Figure 9(d). The standard $\mathrm{BTBF}$ has no false negatives, so it performs well under condition [Pos $>\mathrm{NEG}$ ]. Thus, penalty reductions are more pronounced under [Pos $\approx \mathrm{NeG}$ ]. Posterior Errors stay under 0.035 for all $b p i$, so our posterior expressions are again largely accurate. In this case, as for Uniform streams, Posterior Errors are low for very low $b p i$, where the posterior depends largely on the precisely known priors, and converges to 0.025 when $b p i$ is large.

GBF penalty ratios are shown in Figure 10(c), and Posterior Errors in Figure 10(d). Under [Pos $>\mathrm{NeG}$, the inferential GBF penalty ratio increases for $b p i>5$, since $k$ reaches our maximum 
of 30 by the time $b p i=5$ (see Figure 8 ). After this point, the most effective choice of $k \leq 30$ for the inferential GBF is $k=1$, which incurs lower posterior error, but slightly increases the penalty ratio.

Figures $11(\mathrm{c})$ and $11(\mathrm{~d})$ show that the inferential BTBF has lowest penalty until $b p i \approx 16$ for $[\mathrm{Pos} \approx \mathrm{NeG}$ ], and $b p i \approx 22$ for [Pos $>\mathrm{NeG}$. The inferential BTBF's advantage is greater for $[\mathrm{Pos} \approx \mathrm{NEG}]$ since the standard BTBF has no false negatives, and benefits when most queries return Pos.

6.4.2 Source IP Data Stream. The Source IP data stream [4] draws anonymized source addresses from IPv4 packet headers $\left(|U|=2^{32}\right)$. Address distribution is complex, so $p_{x}$ is hard to model analytically (see Assumption 1). We handled this problem by pre-processing the stream items $x$ to be queried, computing $p_{x}$ based on the observed frequency of address $x$, and saving $\left(x, p_{x}\right)$ pairs for the queried $x$.

We sample $D(j)$ for $j \in\left\{1,10,10^{2}, 10^{3}, 10^{4}, 10^{5}, 2^{18}\right\}$ over the stream itself. We inserted $2^{18}$ items during each of 8 sampling trials. We then averaged $D(j)$ values over all 8 trials, and interpolated between averages using Equation (18).

BTBF penalty ratios are shown in Figure 9(e), and Posterior Errors in Figure 9(f). GBF penalty ratios are in Figure 10(e), and Posterior Errors in Figure 10(f). The stream is bursty, so $p_{x}$ is not strictly time-invariant, violating Assumption 2. Thus, priors are not accurate, leading to higher Posterior Error for low $b p i$, where the posterior relies heavily on the prior.

These high errors, combined with the zero false-negative rate of the standard BTBF, cause the inferential BTBF to incur higher penalties for some low bpi under [Pos $>$ NEG]. However, the inferential BTBF still generally reduces penalties for most trials.

Figures 11(e) and 11(f) show total penalties for the IP Source stream. Every queried item is reinserted. For $[\mathrm{Pos} \approx \mathrm{NEG}$ ], where data is bursty and $w$ is small, any other prior insertions of $x$ are likely to have been recent. The BTBF spends comparable space on each item in the window, but the GBF and Simple Buffer devote more space to more recent items, so they initially outperform the BTBF. The GBF thus has the potential to outperform the BTBF in such scenarios, where more recent items are more important to remember.

Under [Pos $>\mathrm{NEG}$ ], $w$ is larger, so an item is more likely to have multiple bursts throughout the window, which the BTBF handles well. The inferential GBF costs increase near $b p i=9$, where $k$ reaches 30 (Figure 8).

\section{RELATED WORK}

An extensive survey of various Bloom Filter variants appears in Reference [20], the variants being compared with respect to their performance and generality. Applications of Bloom Filters are discussed in Reference [3].

Filters including the Standard Bloom Filter [2], the Generalized Bloom Filter [16], and others $[17,19]$ use single-bit cells. Other filters use multiple bits in each cell to represent counters, as in the Counting Bloom Filter (CBF) [11], timers, as in the TBF [38], or other values [5, 8, 30].

Simple filters $[2,9,31]$ only allow items to be inserted and generally represent static sets. Deletable filters $[19,29,30]$ allow items to be deleted as well as inserted and represent dynamic sets. Decaying filters represent a dynamic set of recently inserted items. As new items are inserted, decaying filters lose their memory of older items.

Deletable filters such as the CBF can function as decaying filters by storing a queue of recent items $[35,36]$. When a new item arrives, an old item is removed from the queue and deleted from the filter. Storing the queue requires many bits per item, so such techniques are only practical when a great deal of space is available to the filter. 
Common decaying filters use multi-bit counters and insert an item by setting all its touched cells to some maximum value such as a window width. Cells are regularly decremented, with minimum value 0 . When the filter is queried, the item is deemed to be in the window if all touched cells have values greater than 0 . In Reference [8], cells to decrement are chosen randomly after each insertion, while in References [15, 33, 39], all non-zero counters are decremented after each block of inserts. The TBF [38] implicitly decrements cells by assigning each cell a timestamp, and periodically incrementing a current timestamp. Posterior expressions for such decaying filters are similar to those of the BTBF.

The work in Reference [14] addresses the false-positive problem by applying techniques from Combinatorial Group Testing to create an auxiliary data structure called the EGH filter, and guarantees the absence of false positives as long as the number of inserted items is below a threshold $d$. Bloom Filters have been used for comparing sets, in applications such as distributed joins [18, 22, 26, 27] and cache management [11]. The work in Reference [21] designs a new data structure called the Invertible Counting Bloom Filter that permits comparison operations, such as set differencing, on multisets.

Current designs ignore much of the information latent in filters. Authors of Reference [15] note that in decaying filters, the number of timers with minimum value touched by $x$ affects the posterior probability that $x$ is in the window, but do not derive that probability. Authors of Reference [31] use specific counter values in a CBF to derive the posterior probability that $x$ is in a static set. They show that the posterior depends on the product of the counters touched by $x$, and use it to improve accuracy. Authors of Reference [5] use knowledge of data stream item frequencies to improve accuracy. They build a hierarchy of decaying filters and assign items to filters based on frequency, using more information to store more frequent items.

We introduced our framework for constructing inferential time-decaying filters and an analysis of the Timing Bloom Filter in Reference [7]. The present work significantly extends and generalizes this prior work in numerous ways. First, it discusses the Generalized Bloom Filter (GBF) and presents an Inferential version of the GBF, with detailed analysis and proofs that analyze its performance and inferential properties. Next, it presents a baseline comparison standard for Bloom filters in the form of an idealized Simple Inferential Buffer approach that uses equivalent total storage, to contrast with the Bloom filter approach of dedicating a fixed number of bits to a small hash for each item in the filter. This comparison is important, because when any Bloom filter variant presented outperforms this approach, it is guaranteed to outperform inferential versions of all such list-based techniques. The current work also presents a direct comparison (total penalty vs. bits per item) between the Inferential BTBF, the Inferential GBF, the Inferential Buffer, and a scheme relying only on prior probabilities ( 0 bits stored per item) as a lower bound on quality. The experiments and implementation presented are greatly expanded, including tables with time per query for each type of filter in microseconds. Finally, this article presents significantly expanded details on optimal parameter selection (Figure 8).

\section{CONCLUSION}

We have shown how to turn standard time-decaying filters into inferential filters, using prior probabilities and previously unused information in the filter. We showed how inferential filters can support new types of retrospective queries and adapt to query-specific error penalties on existing sliding window queries. We developed a space-efficient extension of the existing Timing Bloom Filter called the Block Timing Bloom Filter (BTBF), and turned the standard BTBF into an inferential BTBF. We also developed an inferential version of the Generalized Bloom Filter (GBF).

We showed that our sliding window posterior expressions for the inferential GBF and BTBF are accurate in practice. We experimentally evaluated the standard and inferential filters, 
comparing total penalties incurred by each when answering sliding window queries with queryspecific penalties. The inferential BTBF generally reduced penalties by $10 \%-70 \%$. Accurate modeling of filters and item probabilities is important, as poor modeling can cause inferential filters to perform poorly.

We showed that in most cases, the inferential GBF and BTBF outperform the standard GBF and BTBF when false-positive/false-negative costs vary between queries. We also showed that the inferential BTBF outperforms the inferential GBF when the window width is fixed for all queries, and that the inferential BTBF outperforms buffer-based techniques, such as those using Counting Bloom Filters, when storage space is limited.

Future work in this area may include additional modeling, developing inferential versions of other filters, and identifying optimal parameters for inferential filters.

\section{REFERENCES}

[1] Kursad Asdemir, Özden Yurtseven, and Moin Yahya. 2008. An economic model of click fraud in publisher networks. Int. F. Electron. Commerce 13, 2 (Dec. 2008), 61-90.

[2] B. H. Bloom. 1970. Space/time trade-offs in hash coding with allowable errors. Commun. ACM 13, 7 (1970), $422-426$.

[3] Andrei Broder and Michael Mitzenmacher. 2003. Network applications of Bloom filters: A survey. Internet Math. 1 (Nov. 2003).

[4] CAIDA. 2011. The CAIDA UCSD Anonymized Internet Traces 2011-Equinox Chicago Direction A starting 20110217125904. Retrieved from http://www.caida.org/data/passive/passive_2011_dataset.xml.

[5] K. Cheng, L. Xiang, and M. Iwaihara. 2005. Time-decaying Bloom filters for data streams with skewed distributions. In Proceedings of the RIDE-SDMA 2005. 63-69.

[6] K. Christensen, A. Roginsky, and M. Jimeno. 2010. A new analysis of the false-positive rate of a Bloom filter. Inform. Process. Lett. 110, 21 (2010), 944-949.

[7] Jonathan L. Dautrich Jr. and Chinya V. Ravishankar. 2013. Inferential time-decaying Bloom filters. In Proceedings of the 16th ICDT. ACM, 239-250.

[8] F. Deng and D. Rafiei. 2006. Approximately detecting duplicates for streaming data using stable Bloom filters. In Proceedings of the SIGMOD. 25-36.

[9] B. Donnet, B. Baynat, and T. Friedman. 2006. Retouched Bloom filters: Allowing networked applications to trade off selected false positives against false negatives. In Proceedings of the CoNEXT. 13.

[10] M. Douglas McIlroy. 1982. Development of a spelling list. IEEE Trans. Commun. 1 (Feb. 1982), 91-99.

[11] L. Fan, P. Cao, J. Almeida, and A. Z. Broder. 2000. Summary cache: A scalable wide-area web cache sharing protocol. IEEE/ACM Trans. Netw. 8, 3 (2000), 281-293.

[12] Lee L. Gremillion. 1982. Designing a Bloom filter for differential file access. Commun. ACM 25, 9 (Sep. 1982), 600-604. DOI : https://doi.org/10.1145/358628.358632

[13] D. Guo, J. Wu, H. Chen, Y. Yuan, and X. Luo. 2010. The dynamic Bloom filters. IEEE Trans. Knowl. Data Eng. 22, 1 (2010), 120-133.

[14] Śandor Kiss, Éva Hosszu, János Tapolcai, Lajos Rónyai, and Ori Rottenstreich. 2018. Bloom filter with a false-positivefree zone. In Proceedings of the INFOCOM. IEEE.

[15] G. Koloniari, N. Ntarmos, E. Pitoura, and D. Souravlias. 2011. One is enough: Distributed filtering for duplicate elimination. In Proceedings of the CIKM. 433-442.

[16] R. P. Laufer, P. B. Velloso, and O. C. Duarte. 2011. A generalized Bloom filter to secure distributed network applications. Computer Networks (2011).

[17] X. Li, J. Wu, and J. Xu. 2006. Hint-based routing in WSNs using scope decay Bloom filters. In Proceedings of the IWNAS. IEEE, 8-15.

[18] Zhe Li and Kenneth A. Ross. 1995. PERF join: An alternative to two-way Semijoin and Bloomjoin. In Proceedings of the CIKM. ACM, New York, NY, 137-144. DOI : https://doi.org/10.1145/221270.221360

[19] Y. Lu, B. Prabhakar, and F. Bonomi. 2005. Bloom filters: Design innovations and novel applications. In Proceedings of the Allerton Conference.

[20] Lailong Luo, Deke Guo, Richard T. B. Ma, Ori Rottenstreich, and Xueshan Luo. 2018. Optimizing Bloom filter: Challenges, solutions, and comparisons. CoRR abs/1804.04777. Retrieved from http://arxiv.org/abs/1804.04777.

[21] L. Luo, D. Guo, J. Wu, O. Rottenstreich, Q. He, Y. Qin, and X. Luo. 2017. Efficient multiset synchronization. IEEE/ACM Trans. Netw. 25, 2 (Apr. 2017), 1190-1205. DOI : https://doi.org/10.1109/TNET.2016.2618006 
[22] Lothar F. Mackert and Guy M. Lohman. 1986. $\mathrm{R}^{*}$ optimizer validation and performance evaluation for distributed queries. In Proceedings of the 12th VLD. Morgan Kaufmann Publishers Inc., San Francisco, CA, 149-159. Retrieved from http://dl.acm.org/citation.cfm?id=645913.671480.

[23] Udi Manber and Sun Wu. 1994. An algorithm for approximate membership checking with application to password security. Inf. Process. Lett. 50, 4 (May 1994), 191-197. DOI : https://doi.org/10.1016/0020-0190(94)00032-8

[24] Ahmed Metwally, Dharma Agrawal, and A. El Abbadi. 2005. Duplicate detection in click streams. In Proceedings of the WWW Conference. 12-21.

[25] Calvin N. Mooers. 1951. Zatocoding applied to mechanical organization of knowledge. Amer. Document. 2, 1 (1 1951), 20-32. DOI : https://doi.org/10.1002/asi.5090020107

[26] J. K. Mullin. 1990. Optimal semijoins for distributed database systems. IEEE Trans. Softw. Eng. 16, 5 (May 1990), $558-$ 560. DOI : https://doi.org/10.1109/32.52778

[27] James K. Mullin. 1993. Estimating the size of a relational join. Info. Syst. 18, 3 (Apr. 1993), 189-196. DOI : https://doi. org/10.1016/0306-4379(93)90037-2

[28] L. Page, S. Brin, R. Motwani, and T. Winograd. 1999. The PageRank citation ranking: Bringing order to the web. Technical Report TR 1999-66. Stanford Infolab.

[29] C. E. Rothenberg, C. A. B. Macapuna, F. L. Verdi, and M. F. Magalhaes. 2010. The deletable Bloom filter: A new member of the Bloom family. IEEE Commun. Lett. 14, 6 (2010), 557-559.

[30] O. Rottenstreich, Y. Kanizo, and I. Keslassy. 2012. The variable-increment counting Bloom filter. In Proceedings of the INFOCOM.

[31] O. Rottenstreich and I. Keslassy. 2012. The Bloom paradox: When not to use a Bloom filter? In Proceedings of the INFOCOM.

[32] S. Saroiu, K. P. Gummadi, R. J. Dunn, S. D. Gribble, and H. M. Levy. 2002. An analysis of internet content delivery systems. ACM SIGOPS Operat. Syst. Rev. 36 (2002), 315-327.

[33] H. Shen and Y. Zhang. 2008. Improved approximate detection of duplicates for data streams over sliding windows. 7. Comput. Sci. Technol. 23, 6 (2008), 973-987.

[34] S. Tarkoma, C. Rothenberg, and E. Lagerspetz. 2012. Theory and practice of Bloom filters for distributed systems. IEEE Commun. Surveys Tutor. 99 (2012), 1-25.

[35] X. Wang and H. Shen. 2010. Approximately detecting duplicates for probabilistic data streams over sliding windows. In Proceedings of the PAAP. 263-268.

[36] J. Wei, H. Jiang, K. Zhou, D. Feng, and H. Wang. 2011. Detecting duplicates over sliding windows with RAM-efficient detached counting Bloom filter arrays. In Proceedings of the NAS. 382-391.

[37] W. B. Wu and C. V. Ravishankar. 2003. The performance of difference coding for sets and relational tables. fournal of the ACM ( (JACM) 50, 5 (2003), 665-693.

[38] L. Zhang and Y. Guan. 2008. Detecting click fraud in pay-per-click streams of online advertising networks. In Proceedings of the ICDCS. IEEE, 77-84.

[39] Y. Zhao and J. Wu. 2010. B-SUB: A practical Bloom-filter-based publish-subscribe system for human networks. In Proceedings of the ICDCS. 634-643.

Received June 2018; revised October 2018; accepted October 2018 